\title{
Symplectic duality between complex domains
}

\author{
Antonio J. Di Scala, Andrea Loi, Fabio Zuddas
}

\begin{abstract}
In this paper after extending the definition of symplectic duality (given in 3 for bounded symmetric domains ) to arbitrary complex domains of $\mathbb{C}^{n}$ centered at the origin we generalize some of the results proved in [3] and [4] to those domains.
\end{abstract}

Keywords: Kähler metric; bounded domain; symplectic duality; Hermitian symmetric space.

Subj.Class: 53D05, 58F06.

\section{Introduction}

Let $\left(\mathbb{C} H^{n}, \omega_{h y p}\right)$ be the $n$-dimensional complex hyperbolic space, namely the unit ball in $\mathbb{C}^{n}$ equipped with the Kähler form

$$
\omega_{\text {hyp }}=-\frac{i}{2} \partial \bar{\partial} \log \left(1-\sum_{j=1}^{n}\left|z_{j}\right|^{2}\right)
$$

whose associated Kähler metric is the hyperbolic metric $g_{\text {hyp }}$. It is well-known that $\left(\mathbb{C} H^{n}, \omega_{h y p}\right)$ is globally symplectomorphic to $\left(\mathbb{C}^{n}, \omega_{0}\right)$ where $\omega_{0}=\frac{i}{2} \sum_{j=1}^{n} d z_{j} \wedge d \bar{z}_{j}$ is the standard symplectic form on $\mathbb{C}^{n}=\mathbb{R}^{2 n}$. An explicit diffeomorphism $\Psi_{\text {hyp }}: \mathbb{C} H^{n} \rightarrow \mathbb{C}^{n}$ satisfying

$$
\Psi_{h y p}^{*} \omega_{0}=\omega_{h y p}
$$

is given by:

$$
\Psi_{h y p}(z)=\frac{z}{\sqrt{1-|z|^{2}}}
$$

where $z=\left(z_{1}, \ldots, z_{n}\right)$ and $|z|^{2}=\sum_{k=1}^{n}\left|z_{k}\right|^{2}$. A simple computation shows that the map $\Psi_{\text {hyp }}$ enjoys the following additional property:

$$
\Psi_{\text {hyp }}^{*} \omega_{F S}=\omega_{0},
$$

where we regard $\mathbb{C}^{n}$ as the affine chart $Z_{0} \neq 0$ of the $n$-dimensional complex projective space $\mathbb{C} P^{n}$ endowed with homogeneous coordinates $Z_{0}, \ldots, Z_{n}$ and

$$
\omega_{F S}=\frac{i}{2} \partial \bar{\partial} \log \left(1+\sum_{j=1}^{n}\left|z_{j}\right|^{2}\right), z_{j}=\frac{Z_{j}}{Z_{0}}
$$

is the restriction to $\mathbb{C}^{n} \subset \mathbb{C} P^{n}$ of the Fubini-Study form of $\mathbb{C} P^{n}$.

Properties (2) and (4) have been recently extended in 3. by the first two authors to all bounded symmetric domains $M \subset \mathbb{C}^{n}$ as expressed by the following theorem. Before stating it we recall that to each bounded symmetric domain $M \subset \mathbb{C}^{n}$ endowed with the hyperbolic form $\omega$ (which, in the irreducible case, is a suitable normalization of the Bergman form) one can associate its compact dual $M^{*}$ equipped with the Kähler form $\omega^{*}$ which is given by the pull-back of the Fubini-Study form of $\mathbb{C} P^{N}$ via the Borel-Weil embedding $B W: M^{*} \rightarrow \mathbb{C} P^{N}$, i.e. $B W^{*} \omega_{F S}=\omega^{*}$. Observe that $M^{*}$ can be obtained by a suitable compactification of $\mathbb{C}^{n}$ and the inclusion $\mathbb{C}^{n} \subset M^{*}$ is often referred as the Borel embedding. Notice also that in the case $M=\mathbb{C} H^{n}, M^{*}=\mathbb{C} P^{n}$, the Borel embedding $\mathbb{C}^{n} \subset \mathbb{C} P^{n}$ is the inclusion of the affine chart $Z_{0} \neq 0$ in $\mathbb{C} P^{n}$ and the Borel-Weil embedding $B W: \mathbb{C} P^{n} \rightarrow \mathbb{C} P^{n}$ is the identity map of $\mathbb{C} P^{n}$. 
Theorem 1.1 (Di Scala-Loi [3]) Let $M \subset \mathbb{C}^{n}$ be a bounded symmetric domain endowed with the hyperbolic form $\omega$. Then there exists a symplectic duality, namely a real analytic diffeomorphism $\Psi: M \rightarrow \mathbb{C}^{n}$ sending the origin to the origin and such that:

$$
\begin{aligned}
\Psi^{*} \omega_{0} & =\omega, \\
\Psi^{*} \omega^{*} & =\omega_{0},
\end{aligned}
$$

where $\omega_{0}$ is (the restriction to $M$ of) the flat Kähler form

$$
\omega_{0}=\frac{i}{2} \partial \bar{\partial}|z|^{2}=\frac{i}{2} \sum_{j=1}^{n} d z_{j} \wedge d \bar{z}_{j}
$$

on $\mathbb{C}^{n}$ and where we are denoting by $\omega^{*}$ the restriction of $\omega^{*}$ to $\mathbb{C}^{n}$ via the Borel embedding $\mathbb{C}^{n} \subset M^{*}$. Moreover if $T \subset M$ is a complex and totally geodesic submanifold of $M$ of dimension $k$ then $\Psi(T)=\mathbb{C}^{k}$, i.e. the map $\Psi$ takes complex and totally geodesic submanifolds through the origin of $M$ to complex and totally geodesic submanifolds through the origin of $\mathbb{C}^{n}$ (the latter being equipped with the flat metric).

In order to study to which extents the map $\Psi$ is unique one needs to understand the set of real analytic maps $B: M \rightarrow M$ satisfying $B^{*} \omega_{0}=\omega_{0}$ and $B^{*} \omega=\omega$. In [4], the set of these maps is called the bisymplectomorphism group of the bounded symmetric domain $M$ and is denoted by $\mathcal{B}(M)$. The main result about this group is Theorem 4 in [4. In the case of $\mathbb{C} H^{n}$ this theorem implies the following:

Theorem 1.2 (Di Scala-Loi-Roos [4]) Let $\Psi: \mathbb{C} H^{n} \rightarrow \mathbb{C}^{n}$ be a symplectic duality. Then

$$
\Psi(z)=e^{i g(z)} \Psi_{\text {hyp }}(z) A z,
$$

where $g$ is an arbitrary smooth complex valued function on $\mathbb{C} H^{n}$ depending only on $|z|^{2}, A \in U(n)$ and $\Psi_{\text {hyp }}$ is given by (2) above.

The key ingredient in the proof of the previous theorems is that the dual Kähler form $\omega^{*}$ on $\mathbb{C}^{n}$ can be obtained by the hyperbolic form $\omega$ on $M$ in the following way (see [2] and [10] for details). Since the Kähler form $\omega$ is real analytic and $M$ is contractible one can find a globally defined real analytic Kähler potential $\Phi: M \rightarrow \mathbb{R}$ for $\omega$ around the origin. The potential $\Phi$ can be expanded around the origin as a convergent power series of the variables $z=\left(z_{1}, \ldots, z_{n}\right)$ and $\bar{z}=\left(\bar{z}_{1}, \ldots, \bar{z}_{n}\right)$, where $z$ is the restriction to $M$ of the Euclidean coordinates of $\mathbb{C}^{n}$. By the change of variables $\bar{z} \mapsto-\bar{z}$ in this power series one gets a new power series which is convergent to a global defined and real valued function of $\mathbb{C}^{n}$, denoted by $\Phi(z,-\bar{z})$. It turns out that $\Phi^{*}(z, \bar{z})=-\Phi(z,-\bar{z})$ is a strictly PSH function of $\mathbb{C}^{n}$ and, moreover, $\omega^{*}=\frac{i}{2} \partial \bar{\partial} \Phi^{*}(z, \bar{z})$.

The aim of this paper is to address the problem of extending the previous procedure to an arbitrary $n$-dimensional complex domain $M \subset \mathbb{C}^{n}$ (open, bounded or unbounded connected subset of $\mathbb{C}^{n}$ ) containing the origin $0 \in \mathbb{C}^{n}$. Therefore, we assume that there exists a real analytic strictly PSH function $\Phi: M \rightarrow \mathbb{R}$ on $M$ such that the function $\Phi^{*}(z, \bar{z})=-\Phi(z,-\bar{z})$ is a real valued and strictly PSH function on an open domain $M^{*} \subset \mathbb{C}^{n}$ containing the origin. The pair $\left(M^{*}, \Phi^{*}\right)$ is what we call in this paper a local dual of $(M, \Phi)$. Notice that a local dual is not unique, indeed any neighbourhood of the origin contained in $M^{*}$ is again a dual of $(M, \Phi)$. Observe also that a dual does not exist in general as shown by the following example.

Example 1.3 Consider the two potentials $\Phi_{\text {hyp }}=-\log \left(1-|z|^{2}\right)$ and $\Phi=\Phi_{\text {hyp }}+z+\bar{z}$ for $\omega_{\text {hyp }}$ on the unit disk $\mathbb{C} H^{1} \subset \mathbb{C}$. Then $\Phi$ does not admit a local dual. Indeed the function $\Phi^{*}=\log \left(1+|z|^{2}\right)-z+\bar{z}$ is not a real valued function in any neighbourhood of the origin of $\mathbb{C}$.

Notice that the previous example also shows that the definition of local duality cannot be extended to the case of Kähler forms. Indeed the same Kähler form can have two different potentials one admitting a (local) dual and the other not. Therefore when we speak of local dual of a Kähler form $\omega$ we always assume to have fixed a Kähler potential for it.

Once we have defined a local dual $\left(M^{*}, \Phi^{*}\right)$ of $(M, \Phi)$, we study the analogues of Theorem 1.1 and Theorem 1.2 for the Kähler forms $\omega=\frac{i}{2} \partial \bar{\partial} \Phi$ and $\omega^{*}=\frac{i}{2} \partial \bar{\partial} \Phi^{*}$. More precisely, we say that there exists a (local) $\lambda$-symplectic 
duality between $\omega$ and $\omega^{*}$ if there exist open neighbourhoods of the origin, say $U \subset M$ and $U^{*} \subset M^{*}$, a positive constant $\lambda$ and a diffeomorphism $\Psi: U \rightarrow U^{*}$ such that

$$
\begin{gathered}
\Psi^{*} \omega_{0}=\lambda \omega, \\
\Psi^{*} \lambda \omega^{*}=\omega_{0},
\end{gathered}
$$

where $\omega_{0}$ is the flat Kähler form of $\mathbb{C}^{n}$. If $\lambda=1$ we simply speak of symplectic duality instead of 1-symplectic duality. Therefore the existence of a $\lambda$-symplectic duality between $\omega$ and $\omega^{*}$ is equivalent to that of a local symplectic duality between $\lambda \omega$ and $\lambda \omega^{*}$ (notice that we are not assuming $\Psi(0)=0$ ).

The presence of the constant $\lambda$ in the previous equations is due to the fact that we want to include in our definition also those symplectic forms which do not admit a symplectic duality but for which there exists a $\lambda$-symplectic duality as shown in the following simple example.

Example 1.4 Let $\mu$ be a positive constant, $\mu \neq 1$ and let $\Phi=\mu \Phi_{\text {hyp }}$, with $\Phi_{\text {hyp }}$ as in the previous example. Then the dual of $\Phi$ is $\Phi^{*}=\mu \log \left(1+|z|^{2}\right)$ (defined on $\mathbb{C}$ ). Then it is not hard to see that there exists a $\lambda$-symplectic duality between $\left(\mathbb{C} H^{1}, \Phi_{\text {hyp }}\right)$ and $\left(\mathbb{C}, \Phi^{*}\right)$ if and only if $\lambda \mu=1$. Therefore, even if it does not exist a symplectic duality between $\omega=\frac{i}{2} \partial \bar{\partial} \Phi$ and $\omega^{*}=\frac{i}{2} \partial \bar{\partial} \Phi^{*}$ there exists a $\lambda$-symplectic duality (with $\lambda=\frac{1}{\mu}$ ) between them, given, for example, by the map (3) (with $n=1$ ).

Assumption Throughout all this paper, to avoid triviality, we will assume that the form $\omega$ is not proportional to $\omega_{0}$. This means it cannot exist any open subset of $M$ and a real number $c$ such that $\omega=c \omega_{0}$ on this open set. In fact in this case $\omega^{*}=\omega$ and the existence of a $\lambda$-symplectic duality is equivalent to a single equation $\Psi^{*} \omega_{0}=\omega_{0}$ which is easily solved by taking $\Psi=\mathrm{Id}$. It is worth pointing out that by Darboux's theorem each of the equations (8) and (9) can be separately solved (locally). With the assumption of non proportionality a $\lambda$-symplectic duality $\Psi$ turns out to be a simultaneous symplectomorphism with respect to different symplectic structures, namely $\lambda \omega$ and $\omega_{0}$ on $U$ and $\omega_{0}$ and $\lambda \omega^{*}$ on $U^{*}$. This phenomenon could be of some interest from the physical point of view. Indeed, roughly speaking, it is telling us that the Darboux's coordinates for $\lambda \omega$ are "the inverse" of those of $\lambda \omega^{*}$. Moreover the existence of a $\lambda$-symplectic duality could give strong restrictions on the curvature of the Kähler metric $\omega$ (cf. Section 4 below).

A very interesting case we consider in this paper is that of rotation invariant potentials, and, in particular, radial potentials, namely those $\Phi: M \rightarrow \mathbb{R}$ which depend only on $\left|z_{1}\right|^{2}, \ldots,\left|z_{n}\right|^{2}$ and, in the radial case, on $r=$ $\left|z_{1}\right|^{2}+\cdots+\left|z_{n}\right|^{2}$. Many interesting and important examples of Kähler forms on complex domains are rotation invariant, since they often arise from solutions of ordinary differential equations on the variable $r$ (cf. e.g. 1] and [11). In the rotation invariant case it is easy to see that the local dual $\left(M^{*}, \Phi^{*}\right)$ of $(M, \Phi)$ can be defined (namely $\Phi^{*}$ is real valued and strictly PSH in a suitable neighborhood $M^{*}$ of the origin) and $\Phi^{*}$ is rotation invariant.

The main result of the present paper about $\lambda$-symplectic duality in the rotation invariant case is the following theorem which provides necessary and sufficient conditions for the existence of a special $\lambda$-symplectic duality solely in terms of the potential $\Phi$ (see the beginning of next section for the definition of special map and for the terms involved in the statement of the theorem).

Theorem 1.5 Let $M \subset \mathbb{C}^{n}$ be a complex domain containing the origin endowed with a rotation invariant Kähler potential $\Phi$. Let $\Phi^{*}$ be the dual defined on $M^{*}$ There exists a special $\lambda$-symplectic duality $\Psi: U \rightarrow U^{*}$ between $\omega=\frac{i}{2} \partial \bar{\partial} \Phi$ and $\omega^{*}=\frac{i}{2} \partial \bar{\partial} \Phi^{*}$ (where $U \subset \mathbb{C}^{n}$ and $U^{*} \subset \mathbb{C}^{n}$ are open subsets centered at the origin) if and only if the following equations are satisfied:

$$
\lambda^{2} \frac{\partial \tilde{\Phi}}{\partial x_{k}}\left(x_{1}, \ldots, x_{n}\right) \cdot \frac{\partial \tilde{\Phi}}{\partial x_{k}}\left(-\lambda \frac{\partial \tilde{\Phi}}{\partial x_{1}} x_{1}, \ldots,-\lambda \frac{\partial \tilde{\Phi}}{\partial x_{n}} x_{n}\right)=1, \quad k=1, \ldots, n,
$$

on an open neighbourhood of the origin of $\mathbb{R}^{n}$ contained in $\tilde{M}$. Here $\tilde{\Phi}$ (resp. $\tilde{M}$ ) is the function (resp. the domain) associated to $\Phi$ (resp. $M$ ). Moreover $\Psi$ is uniquely determined by $\Phi$ and it is rotation invariant.

The authors believe it is an interesting and very challenging problem to classify all the $\lambda$-symplectic dualities $\Psi$ in the rotation invariant case without assuming that $\Psi$ is special.

In the radial case we have a complete classification of $\lambda$-symplectic dualities as expressed by the following theorem which can be considered a generalization of Theorem 1.2 above to all radial domains in $\mathbb{C}^{n}$ centered at the origin. 
Theorem 1.6 Let $M \subset \mathbb{C}^{n}$ be a complex domain containing the origin endowed with a radial Kähler potential $\Phi$. Let $\Psi: U \rightarrow U^{*}$ be a $\lambda$-symplectic duality between $\omega=\frac{i}{2} \partial \bar{\partial} \Phi$ and $\omega^{*}=\frac{i}{2} \partial \bar{\partial} \Phi^{*}$. Then there exist an open subset $V \subset U$, containing the origin, a radial function $g: V \rightarrow \mathbb{R}$ and a unitary $n \times n$ matrix $A \in U(n)$ such that

$$
\Psi(z)=e^{i g(z)} \psi(z) A(z), z \in V,
$$

where $\psi: V \rightarrow \mathbb{R}$ is the radial and real-analytic function on $V$ given by

$$
\psi(z)=\left(\lambda f^{\prime}(x)\right)^{\frac{1}{2}}, x=|z|^{2}=\left|z_{1}\right|^{2}+\cdots+\left|z_{n}\right|^{2}
$$

and where $f: \hat{M} \rightarrow \mathbb{R}$ is the function associated to $\Phi$ and $\hat{M}$ is the domain associated to $M$ (see Section (3)).

Consequently there exists a $\lambda$-symplectic duality between $\omega$ and $\omega^{*}$ if and only if

$$
\lambda^{2} f^{\prime}(x) f^{\prime}\left(-\lambda x f^{\prime}(x)\right)=1,
$$

on an open neighbourhood of the origin of $\mathbb{R}$ contained in $\hat{M}$.

The paper is organized as follows. The next two sections (Section 2 and Section 3) are dedicated to the proofs of Theorem 1.5 and Theorem 1.6 respectively, In Section 4 we describe some applications and examples of our results. The paper ends with an appendix containing a technical lemma which is a key ingredient in the proof of our theorems. This lemma is indeed a simple corollary of the results developed in [1] for special symplectic maps. We have included it here to make this paper self-contained as much as possible.

\section{The proof of Theorem 1.5}

Let $M \subset \mathbb{C}^{n}$ be a complex domain containing the origin and let $\Phi$ be a rotation invariant Kähler potential. This means that there exists $\tilde{\Phi}: \tilde{M} \rightarrow \mathbb{R}$, defined on the open subset $\tilde{M} \subset \mathbb{R}^{n}$ given by

$$
\tilde{M}=\left\{x=\left.\left(x_{1}, \ldots, x_{n}\right) \in \mathbb{R}^{n}\left|x_{j}=\right| z_{j}\right|^{2}, z=\left(z_{1}, \ldots, z_{n}\right) \in M\right\}
$$

such that $\Phi(z)=\tilde{\Phi}(x)$. The function $\tilde{\Phi}$ (resp. $\tilde{M}$ ) will be called the function (resp. the domain) associated to $\Phi$ (resp. M). A real analytic map (not necessarily a diffeomorphism) $\Psi: C \rightarrow S: z=\left(z_{1}, \ldots, z_{n}\right) \mapsto\left(\Psi_{1}(z), \ldots, \Psi_{n}(z)\right)$, between two complex domains $C \subseteq \mathbb{C}^{n}$ and $S \subseteq \mathbb{C}^{n}$ containing the origin is said to be special if $\Psi_{j}(z)=\psi_{j}(z) z_{j}, j=$ $1, \ldots, n$ where $\psi_{j}, j=1, \ldots, n$, are real valued functions defined on $C$. We say that a special map $\Psi: C \rightarrow S: z \mapsto$

$\left(\Psi_{1}(z)=\psi_{1}(z) z_{1}, \ldots, \Psi_{n}(z)=\psi_{n}(z) z_{n}\right)$ is rotation invariant if there exist real valued functions $\tilde{\psi}_{j}: \tilde{C} \mapsto \mathbb{R}$, which we call the functions associated to $\Psi$, such that $\tilde{\psi}_{j}(x)=\psi_{j}(z)$ for $x=\left(x_{1}, \ldots, x_{n}\right) \in \tilde{C}, x_{j}=\left|z_{j}\right|^{2}$.

We now prove Theorem 1.5.

Proof of Theorem 1.5; We start by proving the last part of the theorem, namely that a $\lambda$-symplectic duality which is special is necessarily rotation invariant. Actually we will show it for the special maps satisfying only the first equation (8) defining a $\lambda$-symplectic duality, namely $\Psi^{*} \omega_{0}=\lambda \omega$. We can assume $\lambda=1$, namely $\Psi^{*} \omega_{0}=\omega$. In fact the proof extends easily to arbitrary $\lambda$. Notice that $\omega=\frac{i}{2} \sum_{k, l=1}^{n}\left(\frac{\partial^{2} \tilde{\Phi}}{\partial x_{k} \partial x_{l}} \bar{z}_{l} z_{k}+\frac{\partial \tilde{\Phi}}{\partial x_{k}} \delta_{k l}\right) d z_{l} \wedge d \overline{z_{k}}$, where, with a slight abuse of notation, we are omitting the fact that the previous expression has to be evaluated at $x_{1}=\left|z_{1}\right|^{2}, \ldots, x_{n}=\left|z_{n}\right|^{2}$. Hence equation $\Psi^{*} \omega_{0}=\omega$ reads

$$
\sum_{j=1}^{n} d \Psi_{j} \wedge d \bar{\Psi}_{j}=\sum_{k, l=1}^{n}\left(\frac{\partial^{2} \tilde{\Phi}}{\partial x_{k} \partial x_{l}} \bar{z}_{l} z_{k}+\frac{\partial \tilde{\Phi}}{\partial x_{k}} \delta_{k l}\right) d z_{l} \wedge d \overline{z_{k}} .
$$

By comparing the $(1,1),(2,0),(0,2)$ components of the right-hand side and the left-hand side in this equality we get, for every $k, m=1, \ldots, n$,

$$
\sum_{j=1}^{n} \frac{\partial \Psi_{j}}{\partial z_{k}} \frac{\partial \bar{\Psi}_{j}}{\partial z_{m}}=\sum_{j=1}^{n} \frac{\partial \Psi_{j}}{\partial z_{m}} \frac{\partial \bar{\Psi}_{j}}{\partial z_{k}}
$$

and 


$$
\sum_{j=1}^{n}\left[\frac{\partial \Psi_{j}}{\partial z_{k}} \frac{\partial \bar{\Psi}_{j}}{\partial \bar{z}_{m}}-\frac{\partial \bar{\Psi}_{j}}{\partial z_{k}} \frac{\partial \Psi_{j}}{\partial \bar{z}_{m}}\right]=\frac{\partial^{2} \tilde{\Phi}}{\partial x_{k} \partial x_{m}} \bar{z}_{k} z_{m}+\frac{\partial \tilde{\Phi}}{\partial x_{m}} \delta_{k m} .
$$

By inserting $\Psi_{j}=\psi_{j} z_{j}\left(\right.$ and $\bar{\Psi}_{j}=\psi_{j} \bar{z}_{j}$ ) into equations (16) and (17) we get respectively

$$
\begin{gathered}
\frac{\partial \psi_{k}}{\partial z_{m}} \psi_{k} \bar{z}_{k}+\sum_{j=1}^{n} \frac{\partial \psi_{j}}{\partial z_{k}} \frac{\partial \psi_{j}}{\partial z_{m}}\left|z_{j}\right|^{2}=\frac{\partial \psi_{m}}{\partial z_{k}} \psi_{m} \bar{z}_{m}+\sum_{j=1}^{n} \frac{\partial \psi_{j}}{\partial z_{m}} \frac{\partial \psi_{j}}{\partial z_{k}}\left|z_{j}\right|^{2} \\
\frac{\partial \psi_{m}}{\partial z_{k}} \psi_{m} z_{m}+\frac{\partial \psi_{k}}{\partial \bar{z}_{m}} \psi_{k} \bar{z}_{k}+\psi_{k}^{2} \delta_{k m}=\frac{\partial^{2} \tilde{\Phi}}{\partial x_{k} \partial x_{m}} \bar{z}_{k} z_{m}+\frac{\partial \tilde{\Phi}}{\partial x_{m}} \delta_{k m}
\end{gathered}
$$

which can be rewritten as

$$
\frac{1}{2} \frac{\partial \psi_{k}^{2}}{\partial z_{m}} \bar{z}_{k}+\sum_{j=1}^{n} \frac{\partial \psi_{j}}{\partial z_{k}} \frac{\partial \psi_{j}}{\partial z_{m}}\left|z_{j}\right|^{2}=\frac{1}{2} \frac{\partial \psi_{m}^{2}}{\partial z_{k}} \bar{z}_{m}+\sum_{j=1}^{n} \frac{\partial \psi_{j}}{\partial z_{m}} \frac{\partial \psi_{j}}{\partial z_{k}}\left|z_{j}\right|^{2}
$$

and

$$
\frac{1}{2} \frac{\partial \psi_{m}^{2}}{\partial z_{k}} z_{m}+\frac{1}{2} \frac{\partial \psi_{k}^{2}}{\partial \bar{z}_{m}} \bar{z}_{k}+\psi_{k}^{2} \delta_{k m}=\frac{\partial^{2} \tilde{\Phi}}{\partial x_{k} \partial x_{m}} \bar{z}_{k} z_{m}+\frac{\partial \tilde{\Phi}}{\partial x_{m}} \delta_{k m}
$$

If we distinguish in equation (21) the cases $m=k$ and $m \neq k$ we get respectively

$$
\begin{gathered}
R e\left(\frac{\partial \psi_{k}^{2}}{\partial z_{k}} z_{k}\right)=G_{k}-\psi_{k}^{2} \quad k=1, \ldots, n \\
\frac{1}{2} \frac{\partial \psi_{m}^{2}}{\partial z_{k}} z_{m}+\frac{1}{2} \frac{\partial \psi_{k}^{2}}{\partial \bar{z}_{m}} \bar{z}_{k}=\frac{\partial^{2} \tilde{\Phi}}{\partial x_{k} \partial x_{m}} \bar{z}_{k} z_{m}
\end{gathered}
$$

where $G_{k}=\frac{\partial^{2} \tilde{\Phi}}{\partial x_{k}^{2}}\left|z_{k}\right|^{2}+\frac{\partial \tilde{\Phi}}{\partial x_{k}}$ is a rotation invariant function.

Equation (20) implies that $\frac{\partial \psi_{m}^{2}}{\partial z_{k}} \bar{z}_{m}$ is symmetric in $k, m$. So if we multiply equation (23) by $\bar{z}_{m}$, assume $z_{k} \neq 0$ and divide by $\bar{z}_{k}$ we can rewrite it as

$$
\operatorname{Re}\left(\frac{\partial \psi_{k}^{2}}{\partial z_{m}} z_{m}\right)=H_{k m} \quad(k \neq m) .
$$

Up to changing the order of variables, we can assume $k=1$. Let us set $\psi_{1}^{2}=F$. Equations (22) and (24) can be written then as

$$
\begin{gathered}
\operatorname{Re}\left(\frac{\partial F}{\partial z_{1}} z_{1}\right)=G-F \\
\operatorname{Re}\left(\frac{\partial F}{\partial z_{m}} z_{m}\right)=H_{m} \quad(m \neq 1),
\end{gathered}
$$

where we have set $G_{1}=G$ and $H_{1 m}=H_{m}$. So we need to show that the real analytic function $F$ is rotation invariant ( $F$ is real analytic since by definition a special map is real analytic). We will prove that

$$
\frac{\partial^{i_{1}+\ldots+i_{n}+j_{1}+\ldots+j_{n}} F}{\partial z_{1}^{i_{1}} \ldots \partial z_{n}^{i_{n}} \partial \bar{z}_{1}^{j_{1}} \ldots \partial \bar{z}_{n}^{j_{n}}}(0)=0
$$

whenever $\left(i_{1}, \ldots, i_{n}\right) \neq\left(j_{1}, \ldots, j_{n}\right)$. Let us assume first that $i_{k} \neq j_{k}$, where $k \neq 1$. Without loss of generality we can assume that $i_{k}>j_{k}$ (otherwise we conjugate the derivative). Notice that equation (25) can be rewritten as

$$
F=G-\frac{1}{2} \frac{\partial F}{\partial z_{1}} z_{1}-\frac{1}{2} \frac{\partial F}{\partial \bar{z}_{1}} \bar{z}_{1}
$$

Since $G$ is rotation invariant, we get 


$$
\frac{\partial^{i_{k}} F}{\partial z_{k}^{i_{k}}}=\frac{\partial^{i_{k}} G}{\partial x_{k}^{i_{k}}} \bar{z}_{k}^{i_{k}}-\frac{1}{2} \frac{\partial^{i_{k}+1} F}{\partial z_{1} \partial z_{k}^{i_{k}}} z_{1}-\frac{1}{2} \frac{\partial^{i_{k}+1} F}{\partial \bar{z}_{1} \partial z_{k}^{i_{k}}} \bar{z}_{1}
$$

and then, since $j_{k}<i_{k}$

$$
\frac{\partial^{i_{k}+j_{k}} F}{\partial \bar{z}_{k}^{j_{k}} \partial z_{k}^{i_{k}}}=R \bar{z}_{k}-\frac{1}{2} \frac{\partial^{i_{k}+j_{k}+1} F}{\partial z_{1} \partial \bar{z}_{k}^{j_{k}} \partial z_{k}^{i_{k}}} z_{1}-\frac{1}{2} \frac{\partial^{i_{k}+j_{k}+1} F}{\partial \bar{z}_{1} \partial \bar{z}_{k}^{j_{k}} \partial z_{k}^{i_{k}}} \bar{z}_{1}
$$

for some function $R$. By deriving equation (29) with respect to variables different from $z_{1}, \bar{z}_{1}, z_{k}, \bar{z}_{k}$, it is clear that the right-hand side writes as a sum of the kind $A \bar{z}_{k}+B z_{1}+C \bar{z}_{1}$ and then vanishes when evaluated in $z=0$. On the other hand, if we derive the equation with respect to $z_{1}$ (the case $\bar{z}_{1}$ is analogous), then the right-hand side of (29) becomes

$$
\frac{\partial R}{\partial z_{1}} \bar{z}_{k}-\frac{1}{2} \frac{\partial^{i_{k}+j_{k}+1} F}{\partial z_{1} \partial \bar{z}_{k}^{j_{k}} \partial z_{k}^{i_{k}}}-\frac{1}{2} \frac{\partial^{i_{k}+j_{k}+2} F}{\partial z_{1}^{2} \partial \bar{z}_{k}^{j_{k}} \partial z_{k}^{i_{k}}} z_{1}-\frac{1}{2} \frac{\partial^{i_{k}+j_{k}+2} F}{\partial z_{1} \partial \bar{z}_{1} \partial \bar{z}_{k}^{j_{k}} \partial z_{k}^{i_{k}}} \bar{z}_{1} .
$$

so that equation rewrites as

$$
\frac{3}{2} \frac{\partial^{i_{k}+j_{k}+1} F}{\partial z_{1} \partial \bar{z}_{k}^{j_{k}} \partial z_{k}^{i_{k}}}=\frac{\partial R}{\partial z_{1}} \bar{z}_{k}-\frac{1}{2} \frac{\partial^{i_{k}+j_{k}+2} F}{\partial z_{1}^{2} \partial \bar{z}_{k}^{j_{k}} \partial z_{k}^{i_{k}}} z_{1}-\frac{1}{2} \frac{\partial^{i_{k}+j_{k}+2} F}{\partial z_{1} \partial \bar{z}_{1} \partial{\overline{z_{k}}}^{j_{k}} \partial z_{k}^{i_{k}}} \bar{z}_{1} .
$$

In general, deriving $p$ times with respect to $z_{1}$ and $q$ times with respect to $\bar{z}_{1}$ the equation writes as follows

$$
c \frac{\partial^{i_{k}+j_{k}+p+q} F}{\partial z_{1}^{p} \partial \bar{z}_{1}^{q} \partial \bar{z}_{k}^{j_{k}} \partial z_{k}^{i_{k}}}=A \bar{z}_{k}+B z_{1}+C \bar{z}_{1},
$$

for some $c>0$ and some functions $A, B, C$. Then by deriving again this expression with respect to those variables different from $z_{1}, \bar{z}_{1}, z_{k}, \bar{z}_{k}$ and evaluating in $z=0$, it vanishes. In the case $i_{1}>j_{1}$, just derive equation (27) first $i_{1}$ times with respect to $z_{1}, j_{1}$ times with respect to $\bar{z}_{1}$ and apply arguments similar to the above in order to prove that the partial derivative vanishes at $z=0$.

Assume now that there exists a special $\lambda$-symplectic duality $\Psi: U \subset M \rightarrow U^{*} \subset M^{*}$ between $\omega$ and $\omega^{*}$. Then, by what we showed $\Psi$ is rotation invariant. By applying Lemma 5.1 in the Appendix at the end of the paper to $C=U$ and $S=U^{*}$ equipped first with the potentials $\alpha=\lambda \Phi$ and $\beta=|z|^{2}$ and then with the potentials $\alpha=|z|^{2}$ and $\beta=\lambda \Phi^{*}$ one gets that (8) and (9) are equivalent to the following equations on $\tilde{U}$ (the open set associated to $U$ ):

$$
\left\{\begin{array}{l}
\tilde{\psi}_{k}^{2}=\lambda \frac{\partial \tilde{\Phi}}{\partial x_{k}} \\
\tilde{\psi}_{k}^{2} \cdot \lambda \frac{\partial \tilde{\Phi}^{*}}{\partial x_{k}}\left(\tilde{\psi}_{1}^{2} x_{1}, \ldots, \tilde{\psi}_{n}^{2} x_{n}\right)=1, k=1, \ldots, n .
\end{array}\right.
$$

Observe now that, by the very definition of duality, one has $\tilde{\Phi}^{*}(x)=-\tilde{\Phi}(-x)$ and so $\frac{\partial \tilde{\Phi}^{*}}{\partial x_{k}}(x)=\frac{\partial \tilde{\Phi}}{\partial x_{k}}(-x)$. Therefore equations (33) are equivalent to the following:

$$
\left\{\begin{array}{l}
\tilde{\psi}_{k}^{2}=\lambda \frac{\partial \tilde{\Phi}}{\partial x_{k}} \\
\tilde{\psi}_{k}^{2} \cdot \lambda \frac{\partial \tilde{\Phi}}{\partial x_{k}}\left(-\tilde{\psi}_{1}^{2} x_{1}, \ldots,-\tilde{\psi}_{n}^{2} x_{n}\right)=1, k=1, \ldots, n .
\end{array}\right.
$$

By inserting the first equation of (34) into the second one we get that (10) is satisfied on $\tilde{U} \subset \tilde{M}$. Conversely, assume (10) holds true on a open neighbourhood of the origin, say $\tilde{W} \subset \tilde{M} \subset \mathbb{R}^{n}$. Since $\Phi$ is a rotation invariant Kähler potential we can assume, by shrinking $\tilde{W}$ if necessary, that the function $\frac{\partial \tilde{\Phi}}{\partial x_{k}}$ is positive on $\tilde{W}$ (cf. formula (15) above at $z=0)$. Hence we can define $\tilde{\psi}_{k}: \tilde{W} \subset \mathbb{R}^{n} \rightarrow \mathbb{R}, k=1, \ldots, n$ by setting

$$
\tilde{\psi}_{k}(x)=\left(\lambda \frac{\partial \tilde{\Phi}}{\partial x_{k}}(x)\right)^{\frac{1}{2}}, x \in \tilde{W} .
$$

It follows by (10) that equations (34) (and hence equations (330) are satisfied on $\tilde{W}$. Hence, again by Lemma 5.1 , the rotation invariant special map $\Psi: W \rightarrow M^{*}: z \mapsto\left(\psi_{1}(z) z_{1}, \ldots, \psi_{n}(z) z_{n}\right)$ defined by $\psi_{j}(z)=\tilde{\psi}_{j}(x)$ (where $W$ is the open set whose associated set is $\tilde{W}$ ) satisfies $\Psi^{*} \omega_{0}=\lambda \omega$ and $\Psi^{*} \lambda \omega^{*}=\omega_{0}$. Since $\Psi$ is a local diffeomorphism sending 
the origin to the origin it follows by the inverse function theorem that there exist open neighbourhoods of the origin $U \subset W \subset M$ and $U^{*} \subset M^{*}$ such that the restriction $\Psi_{\mid U}: U \rightarrow U^{*}$ is a diffeomorphism and hence $\Psi$ is a special $\lambda$-symplectic duality between $\omega$ and $\omega^{*}$. Finally, notice that equation (35) shows that $\Psi$ is uniquely determined by the potential $\Phi$.

\section{The proof of Theorem $\mathbf{1 . 6}$}

Let $M \subset \mathbb{C}^{n}$ be a complex domain containing the origin. Let assume that $\Phi$, the potential of the Kähler form $\omega$ is radial and real analytic. Therefore there exists a real analytic function $f: \hat{M} \rightarrow \mathbb{R}$, defined on $\hat{M}=\left\{\left.x \in \mathbb{R}|x=| z\right|^{2}, z \in M\right\}$ such that $\Phi(z)=f(x)$. The function $f$ (resp. $\hat{M}$ ) will be called the function (resp. the domain) associated to $\Phi$ (resp. $M$ ). In what follows, due to the radiality of $\Phi$, all the neighbourhoods of the origin involved can be taken to be open balls centered at the origin (of a suitable radius).

Before proving Theorem 1.6 we make a remark about it. Notice that the maps $\varphi_{1}$ and $\varphi_{2}$ from $V$ to $V$ given by $\varphi_{1}(z)=A(z), A \in U(n)$ and $\varphi_{2}(z)=e^{i g(z)} z$ where $g$ is an arbitrary radial function on $V$ satisfy $\varphi_{1}^{*} \omega_{0}=\varphi_{2}^{*} \omega_{0}=\omega_{0}$ and $\varphi_{1}^{*} \omega=\varphi_{2}^{*} \omega=\omega$ (the equalities regarding the map $\varphi_{1}$ follow by the $U(n)$-invariance of $\omega_{0}$ and $\omega$ while those regarding $\varphi_{2}$ follow by straightforward computations). Hence Theorem 1.6] is telling us that, in the radial case, a $\lambda$-symplectic duality between $\omega$ and $\omega^{*}$ is uniquely determined, up to the composition with a unitary transformation and to the multiplication with a $S^{1}$-valued radial function, by the special $\lambda$-symplectic duality:

$$
z \mapsto \psi(z) z, \psi(z)=\left(\lambda f^{\prime}(x)\right)^{\frac{1}{2}}, x=|z|^{2} .
$$

Proof of the second part of Theorem 1.6: We start proving the second part of the theorem (namely equation (12) and the fact that equation (13) is equivalent to the existence of a $\lambda$-symplectic duality). So assume that equation (13) is satisfied. Then, by Theorem 1.5 (cfr. formula (35) ) the map $\Psi$ given by (36) is (in a suitable neighbourhood of the origin) a (special) $\lambda$-symplectic duality between $\omega$ and $\omega^{*}$ (this also proves (12)). Conversely, if $\Psi: U \rightarrow U^{*}$ is a $\lambda$-symplectic duality between $\omega$ and $\omega^{*}$, then, by the first part of the theorem, it is of the form (11) in a suitable neighbourhood $V \subset U$ of the origin. Therefore, by the previous remark it exists a special $\lambda$-symplectic duality between $\omega$ and $\omega^{*}$ given by the map (36) and hence equation (13) holds true again by Theorem 1.5 (on a suitable neighbourhood of the origin of $\mathbb{R}$ ).

Proof of the first part of Theorem 1.6.

The proof of the first part of the theorem, namely that a $\lambda$-symplectic duality can be written as (11) is quite involved since we are not assuming $\Psi$ to be special. It is obtained by various steps. The first one deals with the complex one dimensional case.

Step 1. Let $M \subset \mathbb{C}$ be a 1-dimensional complex domain containing the origin. endowed with a radial Kähler potential $\Phi$ and let $\Psi: U \rightarrow U^{*}$ be a $\lambda$-symplectic duality between $\omega=\frac{i}{2} \partial \bar{\partial} \Phi$ and $\omega^{*}=\frac{i}{2} \partial \bar{\partial} \Phi^{*}$. Then there exist radial functions $g: U \rightarrow \mathbb{R}$ and $\psi: U \rightarrow \mathbb{R}$ such that

$$
\Psi(z)=e^{i g(z)} \psi(z) z
$$

Moreover $\psi$ is given by

$$
\psi(z)=\left(\lambda f^{\prime}(x)\right)^{\frac{1}{2}}, x=|z|^{2}
$$

Remark 3.1 Notice that in the one-dimensional case, in contrast to the general case, we are not forced to restrict to $V \subset U$ in order to get (37). It should be possible to give an alternative proof of Theorem 1.6 when $n \geq 2$ (cf. the proof of Step 3 and Step 4 below) where one does not need to shrink $U$ (this is obviously true if $\Psi$ is assumed to be real-analytic). Nevertheless for our purposes this is not really important since in this paper we are interested only on the local behavior of a $\lambda$-symplectic duality.

Proof: Let us assume that $U=D_{a}(0), U^{*}=D_{a^{*}}(0)$, where $a$ and $a^{*}$ are suitable real numbers. Let $(r, \theta)$ (resp. $(\rho, \eta))$ be polar coordinates on $U$ (resp. on $\left.U^{*}\right)$. Then we have

$$
\omega_{0}=r d r \wedge d \theta
$$




$$
\begin{gathered}
\omega=S\left(r^{2}\right) r d r \wedge d \theta \\
\omega^{*}=S\left(-\rho^{2}\right) \rho d \rho \wedge d \eta
\end{gathered}
$$

where we have set $S(x)=\left(x f^{\prime}\right)^{\prime}$. Notice that, by (40), $S>0$ because $\omega$ is a Kähler form. Let $\Psi$ be given in polar coordinates by $\Psi(r, \theta)=(\rho(r, \theta), \eta(r, \theta))$. Then $\Psi^{*} \omega_{0}=\lambda \omega$ writes

$$
\rho\left(\rho_{r} \eta_{\theta}-\rho_{\theta} \eta_{r}\right) d r \wedge d \theta=\lambda S\left(r^{2}\right) r d r \wedge d \theta
$$

and $\Psi^{*} \lambda \omega^{*}=\omega_{0}$ writes

$$
\lambda \rho S\left(-\rho^{2}\right)\left(\rho_{r} \eta_{\theta}-\rho_{\theta} \eta_{r}\right) d r \wedge d \theta=r d r \wedge d \theta .
$$

Let us write these equalities as scalar equations as follows

$$
\begin{gathered}
\rho\left(\rho_{r} \eta_{\theta}-\rho_{\theta} \eta_{r}\right)=\lambda S\left(r^{2}\right) r, \\
\lambda \rho S\left(-\rho^{2}\right)\left(\rho_{r} \eta_{\theta}-\rho_{\theta} \eta_{r}\right)=r .
\end{gathered}
$$

Notice that $\left(\rho_{r} \eta_{\theta}-\rho_{\theta} \eta_{r}\right)$ is the Jacobian determinant $J_{\Psi}$ of $\Psi$, and by (44) we have $J_{\Psi}>0$ (recall that $S>0$ ). If we substitute (44) in (45) we get

$$
S\left(-\rho^{2}\right) S\left(r^{2}\right)=\lambda^{-2} .
$$

If we derive this equation with respect to $\theta$ we get

$$
-2 S^{\prime}\left(-\rho^{2}\right) \rho \rho_{\theta} S\left(r^{2}\right)=0 .
$$

Now, if $\rho_{\theta} \neq 0$ at some point, it does not vanish for $r$ belonging to some open real interval. Then, since $S>0$, it must be $S^{\prime} \equiv 0$ in this interval. But, by (40) this would imply that $\omega$ is proportional to $\omega_{0}$, in contrast with our assumption. We conclude that $\rho_{\theta}=0$, i.e. $\rho$ depends only on $r$.

Moreover, (44) becomes

$$
\rho \rho_{r} \eta_{\theta}=\lambda S\left(r^{2}\right) r
$$

Since $J_{\Psi}=\rho_{r} \eta_{\theta}$ does not vanish, both $\rho_{r}$ and $\eta_{\theta}$ are not zero, so this equation implies that $\rho(0)=0$, that is $\Psi(0)=0$. Now, if we divide (48) by $\rho \rho_{r}$ and integrate we get

$$
\eta=\frac{\lambda S\left(r^{2}\right) r}{\rho \rho_{r}} \theta+c(r)
$$

for some function $c$. Now, let us fix $r_{0}$ and let us consider the map $f: S_{r_{0}}^{1} \rightarrow S_{\rho\left(r_{0}\right)}^{1}, e^{i \theta} \mapsto e^{i \eta}$, induced by $\Psi$ on the circle centered at the origin and of radius $r_{0}$, where $\eta$ is given by (49). On the one hand, the $\operatorname{degree} \operatorname{deg}(f)$ of this map equals 1 because $\Psi$ is an orientation-preserving diffeomorphism $\left(J_{\Psi}>0\right)$, on the other hand we have

$$
\operatorname{deg}(f)=\frac{1}{2 \pi} \int_{0}^{2 \pi} \frac{d \eta}{d \theta} d \theta=\left.\frac{\lambda S\left(r^{2}\right) r}{\rho \rho_{r}}\right|_{r=r_{0}}
$$

so that we get $\frac{\lambda S\left(r^{2}\right) r}{\rho \rho_{r}}=1$ and thus $\eta=\theta+c(r)$. Then

$$
\Psi\left(r e^{i \theta}\right)=\rho(r) e^{i \eta}=\rho(r) e^{i \theta} e^{i c(r)},
$$

which proves (38) for $\psi(z)=\rho(r) / r$ and $g(z)=c(r)$. Finally, formula (38) is exactly (12) (which we have already proved in general) in the one-dimensional case.

Before passing to the general case we pause to obtain additional results needed for the proof. Let $\Phi: M \rightarrow \mathbb{R}$ be a radial potential for $\omega$, let $\omega^{*}$ be its dual sympletic form defined in $M^{*}$ and let $f: \hat{M} \rightarrow \mathbb{R}$ be the function associated to $\Phi$. A simple computation shows that: 


$$
\begin{gathered}
\omega=f^{\prime \prime}\left(|z|^{2}\right) \frac{i}{2}\left(\partial|z|^{2} \wedge \bar{\partial}|z|^{2}\right)+f^{\prime}\left(|z|^{2}\right) \omega_{0} \\
\omega^{*}=-f^{\prime \prime}\left(-|z|^{2}\right) \frac{i}{2}\left(\partial|z|^{2} \wedge \bar{\partial}|z|^{2}\right)+f^{\prime}\left(-|z|^{2}\right) \omega_{0} .
\end{gathered}
$$

Remark 3.2 Notice that the assumption that $\omega$ and $\omega_{0}$ are not proportional made at the beginning of the paper in the radial case simply means that it cannot exists an open interval of $\mathbb{R}$ where $f^{\prime}$ is constant. In particular it cannot exist any constant $c$ such that $x f^{\prime \prime}+f^{\prime}=c$ in some open interval of $\mathbb{R}$.

Given a diffeomorphism $\Psi: U \rightarrow U^{*}$ between open subsets $U, U^{*} \subset \mathbb{C}^{n}$ containing the origin we introduce the operators $B_{z}, B_{z}^{*} \in \operatorname{End}\left(T_{z} U\right)$ as follows:

$$
\omega_{z}(\cdot, \cdot)=\omega_{0}\left(B_{z} \cdot, \cdot\right), \quad \omega_{z}^{*}(\cdot, \cdot)=\omega_{0}\left(B_{z}^{*}, \cdot\right) .
$$

We can compute explicitly both operators $B_{z}, B_{z}^{*}$ by using equations (51) and (52). Namely,

$$
\begin{gathered}
B_{z}=f^{\prime \prime}\left(|z|^{2}\right) z \odot \bar{z}+f^{\prime}\left(|z|^{2}\right) \mathrm{Id} \\
B_{z}^{*}=-f^{\prime \prime}\left(-|z|^{2}\right) z \odot \bar{z}+f^{\prime}\left(-|z|^{2}\right) \mathrm{Id}
\end{gathered}
$$

where

$$
(z \odot \bar{z})(v):=\langle v, z\rangle z=\left(\sum_{j=1}^{n} v_{j} \bar{z}_{j}\right) z
$$

and where $\omega_{0}$ is the flat form, i.e. $\omega_{0}(v, w)=\frac{i}{2} \partial \bar{\partial}|z|^{2}(v, w)=-\operatorname{Im}(\langle v, w\rangle)=-\operatorname{Im}\left(\sum_{j} v_{j} \overline{w_{j}}\right.$ ) (so that $g_{0}(v, w)=$ $\omega_{0}(v, i w)$, and $\left.\langle\cdot, \cdot\rangle=g_{0}-i \omega_{0}\right)$. Notice that both operators $B_{z}$ and $B_{z}^{*}$ satisfy $B_{z}(\mathbb{C} z)=B_{z}^{*}(\mathbb{C} z)=\mathbb{C} z$. Define $d \Psi_{z}^{s}: T_{\Psi(z)} U^{*} \rightarrow T_{z} U$ by the equation

$$
\omega_{0}\left(d \Psi_{z}(v), w\right)=\omega_{0}\left(v, d \Psi_{z}^{s}(w)\right)
$$

for all $z \in U$ and for all $v \in T_{z} U$ and $w \in T_{\Psi(z)} U^{*}$.

We can now translate the $\lambda$-symplectic duality conditions for $\Psi: U \rightarrow U^{*}$ in terms of the previous operators. Indeed, the equations of the symplectic duality give

$$
\begin{gathered}
\omega_{0}\left(d \Psi_{z}(v), d \Psi_{z}(w)\right)=\lambda \omega_{z}(v, w)=\lambda \omega_{0}\left(B_{z} v, w\right), \\
\lambda \omega^{*}\left(d \Psi_{z}(v), d \Psi_{z}(w)\right)=\lambda \omega_{0}\left(B_{\Psi(z)}^{*} d \Psi_{z}(v), d \Psi_{z}(w)\right)=\omega_{0}(v, w),
\end{gathered}
$$

for all $v, w \in T_{z} U$. Then we get respectively:

$$
\begin{gathered}
d \Psi_{z}^{s} \circ d \Psi_{z}=\lambda B_{z} . \\
d \Psi_{z}^{s} \circ B_{\Psi(z)}^{*} \circ d \Psi_{z}=\lambda^{-1} \mathrm{Id}
\end{gathered}
$$

By inserting into (57) the explicit formula of $B_{\Psi(z)}^{*}$ given by (55) we get:

$$
\begin{aligned}
\lambda^{-1} \mathrm{Id} & =d \Psi_{z}^{s} \circ\left(-f^{\prime \prime}\left(-|\Psi(z)|^{2}\right) \Psi(z) \odot \overline{\Psi(z)}+f^{\prime}\left(-|\Psi(z)|^{2}\right) \mathrm{Id}\right) \circ d \Psi_{z}= \\
& =-f^{\prime \prime}\left(-|\Psi(z)|^{2}\right) d \Psi_{z}^{s} \circ \Psi(z) \odot \overline{\Psi(z)} \circ d \Psi_{z}+f^{\prime}\left(-|\Psi(z)|^{2}\right) d \Psi_{z}^{s} \circ d \Psi_{z}
\end{aligned}
$$

By (56), (54):

$$
\begin{aligned}
\lambda^{-1} \mathrm{Id} & =-f^{\prime \prime}\left(-|\Psi(z)|^{2}\right) d \Psi_{z}^{s} \circ \Psi(z) \odot \overline{\Psi(z)} \circ d \Psi_{z}+f^{\prime}\left(-|\Psi(z)|^{2}\right) \lambda B_{z}= \\
& =-f^{\prime \prime}\left(-|\Psi(z)|^{2}\right) d \Psi_{z}^{s} \circ \Psi(z) \odot \overline{\Psi(z)} \circ d \Psi_{z}+\lambda f^{\prime}\left(-|\Psi(z)|^{2}\right) f^{\prime \prime}\left(|z|^{2}\right) z \odot \bar{z}+ \\
& +\lambda f^{\prime}\left(-|\Psi(z)|^{2}\right) f^{\prime}\left(|z|^{2}\right) \operatorname{Id}=
\end{aligned}
$$

Finally, by the very defintion of $\odot$ one gets:

$$
\begin{aligned}
\lambda^{-1} \mathrm{Id} & =-f^{\prime \prime}\left(-|\Psi(z)|^{2}\right) d \Psi_{z}^{s}\left(\left\langle d \Psi_{z}(\cdot), \Psi(z)\right\rangle \Psi(z)\right)+ \\
& +\lambda f^{\prime}\left(-|\Psi(z)|^{2}\right)\left(f^{\prime \prime}\left(|z|^{2}\right) z \odot \bar{z}+f^{\prime}\left(|z|^{2}\right) \mathrm{Id}\right) .
\end{aligned}
$$


We are now ready to continue the proof of the theorem.

Let us come back to the general case. In all the following steps $\Psi: U \rightarrow U^{*}$ is a $\lambda$-symplectic duality between open subsets of $\mathbb{C}^{n}$ with $n \geq 2$.

Step 2. The map $\Psi$ sends the origin to the origin, i.e., $\Psi(0)=0$. Consequently $f^{\prime}(0)=\lambda^{-1}$ and $\omega=\omega^{*}=\lambda^{-1} \omega_{0}$ at the origin $0 \in U \subset \mathbb{C}^{n}$.

Proof: Taking $z=0$ in (56) and (57) and taking into account (54) and (55) one gets:

$$
\begin{gathered}
d \Psi_{0}^{s} \circ d \Psi_{0}=\lambda B_{0}=\lambda f^{\prime}(0) \mathrm{Id} \\
d \Psi_{0}^{s} \circ B_{\Psi(0)}^{*} \circ d \Psi_{0}=\lambda^{-1} \mathrm{Id}
\end{gathered}
$$

which imply $B_{\Psi(0)}^{*}=\frac{\lambda^{-2}}{f^{\prime}(0)}$ Id. This together with (55) gives:

$$
B_{\Psi(0)}^{*}=-f^{\prime \prime}\left(-|\Psi(0)|^{2}\right) \Psi(0) \odot \overline{\Psi(0)}+f^{\prime}\left(-|\Psi(0)|^{2}\right) \operatorname{Id}=\frac{\lambda^{-2}}{f^{\prime}(0)} \operatorname{Id} .
$$

Assume now, by contradiction, that $\Psi(0) \neq 0$. Then the previous equation forces $-f^{\prime \prime}\left(-|\Psi(0)|^{2}\right)=0$ which, together with (52), implies that $\omega^{*}=c \omega_{0}$ at the point $\Psi(0)$, where $c=f^{\prime}\left(-|\Psi(0)|^{2}\right)$. Since both forms $\omega_{0}, \omega^{*}$ are $U(n)$-invariant it follows that $\omega^{*}=c \omega_{0}$ at all points of the sphere centered at the origin of radius $r=|\Psi(0)|$. Since $\Psi$ is a diffeomorphism there exists a non constant smooth curve $\gamma:(-\epsilon, \epsilon) \rightarrow U$ such that $\gamma(0)=0, \delta=|\gamma(\epsilon)|>0$ and $|\Psi(\gamma(t))|=|\Psi(\gamma(0))|=r$, for all $t \in(-\epsilon, \epsilon)$. We claim that $\omega$ and $\omega_{0}$ are proportional inside the ball $D_{\delta}(0)$, i.e. the ball centered at the origin of radius $\delta$. This will give the desired contradiction since we are assuming that $\omega_{0}$ and $\omega$ are not proportional (see Remark 3.2). In order to prove our claim let $\beta=\Psi(\gamma)$ be the image of $\gamma$ under $\Psi$. By construction, the curve $\beta$ is contained in the sphere of radius $r$ centered at the origin. It follows, from the previous discussion, that $\left.\omega^{*}\right|_{\beta}=\left.c \omega_{0}\right|_{\beta}$. This, together with the fact that $\Psi$ is a $\lambda$-symplectic duality, implies (by restriction to the curve $\gamma$ ) that

$$
\left.\left(\Psi^{*} \omega_{0}\right)\right|_{\gamma}=\left.\lambda \omega\right|_{\gamma},\left.\quad\left(\Psi^{*} \lambda \omega^{*}\right)\right|_{\gamma}=\left.\lambda c\left(\Psi^{*} \omega_{0}\right)\right|_{\gamma}=\left.\omega_{0}\right|_{\gamma}
$$

and so $\left.\omega\right|_{\gamma}=\left.\lambda^{-2} c^{-1} \omega_{0}\right|_{\gamma}$. Thus, since both forms $\omega, \omega_{0}$ are $U(n)$-invariant it follows that the above equalities hold for all the points on the sphere centered at zero of radius $|\gamma(t)|$, for all $t \in(-\epsilon, \epsilon)$. Now if $t$ runs from 0 to $\epsilon$ the radius of these spheres runs from 0 to $\delta$. So we get that $\omega$ and $\omega_{0}$ are proportional to each other on $D_{\delta}(0)$, as we claim. The last part of Step 2 is now straightforward. Indeed, since $\Psi(0)=0$ by (59) we get $\left(f^{\prime}(0)\right)^{2}=\lambda^{-2}$ and since $f^{\prime}(0)>0$ (this inequality is a consequence of (51) and the fact that $\omega$ is a Kähler form) it follows that $f^{\prime}(0)=\lambda^{-1}$, which again by (51) and (52) implies $\omega=\omega^{*}=\lambda^{-1} \omega_{0}$ at the origin.

Step 3. There exists an open subset $W \subset U$ containing the origin and a nowhere dense subset $S \subset W$ such that:

$$
\left\langle d \Psi_{z}(\mathbb{C} z), \Psi(z)\right\rangle=\mathbb{C}, \forall z \in W \backslash S,
$$

i.e., for each $z \in W \backslash S$ and $\beta \in \mathbb{C}$ there exists $\alpha \in \mathbb{C}$ such that $\left\langle d \Psi_{z}(\alpha z), \Psi(z)\right\rangle=\beta$.

Proof: Let $\eta=x+i y$ be a complex number. Then $\left\langle d \Psi_{z}(\eta z), \Psi(z)\right\rangle=x a(z)+y b(z)$, where

$$
a(z)=\left\langle d \Psi_{z}(z), \Psi(z)\right\rangle, \quad b(z)=\left\langle d \Psi_{z}(i z), \Psi(z)\right\rangle .
$$

To prove this step we need to find an open subset $W \subset U$ containing the origin and a nowhere dense set $S \subset W$ such that $a(z)$ and $b(z)$ are $\mathbb{R}$-independent on $W \backslash S$. We first show that there exists an open subset $W \subset U$ containing the origin where $b(z) \neq 0$ for all $z \in W \backslash\{0\}$. Indeed, assume, by contradiction, that such a set does not exist. Then there exists a sequence $\left\{z_{n}\right\}, z_{n} \in U, z_{n} \neq 0$, with $z_{n} \rightarrow 0$ as $n$ tends to infinity and such that $b\left(z_{n}\right)=0$ for all $n$. Set $w_{n}=\frac{z_{n}}{\left|z_{n}\right|}$ and $t_{n}=\left|z_{n}\right|$. Then $z_{n}=t_{n} w_{n},\left|w_{n}\right|=1$ and $t_{n} \rightarrow 0$. Without loss of generality, since the unit sphere is compact, we can assume that there exists $\xi \in U,|\xi|=1$, such that $w_{n} \rightarrow \xi$. Therefore

$$
0=b\left(z_{n}\right)=\left\langle t_{n} d \Psi_{t_{n} w_{n}}\left(i w_{n}\right), \Psi\left(t_{n} w_{n}\right)\right\rangle,
$$

for all $n$. Dividing by $t_{n}^{2}$ and taking the limit as $n \rightarrow \infty$ we get,

$$
\left\langle d \Psi_{0}(i \xi), d \Psi_{0}(\xi)\right\rangle=0 .
$$


On the other hand

$$
\begin{aligned}
\operatorname{Im}\left(\left\langle d \Psi_{0}(i \xi), d \Psi_{0}(\xi)\right\rangle\right) & =-\omega_{0}\left(d \Psi_{0}(i \xi), d \Psi_{0}(\xi)\right)=-\left(\Psi^{*} \omega_{0}\right)_{0}(i \xi, \xi) \\
& \left.=-\omega_{0}(i \xi, \xi)=\operatorname{Im}(\langle i \xi, \xi)\rangle\right)=|\xi|^{2}=1
\end{aligned}
$$

which contradicts the previous equality. (The equality $\left(\Psi^{*} \omega_{0}\right)_{0}=\omega_{0}$ follows by $\left(\Psi^{*} \omega_{0}\right)=\lambda \omega$ and the fact that $\omega$ at the origin equals $\lambda^{-1} \omega_{0}$, by Step 2).

Fix now an open set $W$ containing the origin such that $b(z) \neq 0$ for all $z \in W \backslash\{0\}$ and let $S$ be the set of points in $W$ where the functions $a$ and $b$ are $\mathbb{R}$-linearly dependent, i.e. $S$ consists of those $z \in W$ for which there exists a real number $r(z)$ such that $a(z)=r(z) b(z)$. Notice that $0 \in S$. For each $z \in S$ let $X(z)$ be the vector at $z$ defined by

$$
X(z)=(1-i r(z)) z
$$

Then it is immediate to see that

$$
\left\langle d \Psi_{z}(X(z)), \Psi(z)\right\rangle=0, \forall z \in S .
$$

The proof will be completed if we show that the interior of $S$ is empty. Assume the contrary and let $\tilde{S}$ be an open subset contained in $S$. Then (61) gives rise to a smooth vector field $X$ on $\tilde{S}$. By inserting $X(z)$ in both sides of equality (58), using (62) and $(z \odot \bar{z})(X(z))=\langle X(z), z\rangle z=|z|^{2} X(z)$, one gets:

$$
\begin{aligned}
\lambda^{-1} X(z) & =\lambda f^{\prime}\left(-|\Psi(z)|^{2}\right)\left(f^{\prime \prime}\left(|z|^{2}\right) z \odot \bar{z}(X(z))+f^{\prime}\left(|z|^{2}\right) X(z)\right) \\
& =\lambda f^{\prime}\left(-|\Psi(z)|^{2}\right)\left(f^{\prime \prime}\left(|z|^{2}\right)|z|^{2}+f^{\prime}\left(|z|^{2}\right)\right) X(z)
\end{aligned}
$$

which implies

$$
\lambda^{-2}=f^{\prime}\left(-|\Psi(z)|^{2}\right)\left(f^{\prime \prime}\left(|z|^{2}\right)|z|^{2}+f^{\prime}\left(|z|^{2}\right)\right) .
$$

Let now $z(t) \subset \tilde{S}$ be an integral curve of the vector field $X(z)$, where $t$ is varying on an open interval, say $I \subset \mathbb{R}$. Notice that (62) implies that $\frac{\partial|\Psi|^{2}}{\partial X}(z)=0$ for all $z \in \tilde{S}$, and hence $|\Psi(z(t))|^{2}$ is a constant, say $d$, for all $t \in I$. By inserting $z(t)$ in the above equality we then get:

$$
c=f^{\prime \prime}\left(|z(t)|^{2}\right)|z(t)|^{2}+f^{\prime}\left(|z(t)|^{2}\right), t \in I,
$$

where $c=\left(\lambda^{2} f^{\prime}(-d)\right)^{-1}$. On the other hand it follows by the very definition of $X(z)$ that $|z(t)|^{2}$ is not a constant function on $I$. Hence, when $t$ is varying in $I, x=|z(t)|^{2}$ is varying in a non-empty open interval of the real line. In this interval the function $f$ satisfies the differential equation $f^{\prime \prime}(x) x+f^{\prime}(x)=c$ contradicting our assumption (see Remark [3.2).

Step 4. There exists an open subset $V \subset U$ where the following condition is satisfied: given $z \in V$ and $\beta \in \mathbb{C}$ one can find a complex number $\delta$ (depending on $\beta$ and $z$ ) such that $d \Psi_{z}(\beta z)=\delta \Psi(z)$. If this happens we will write

$$
d \Psi_{z}(\mathbb{C} z)=\mathbb{C} \Psi(z), \forall z \in V .
$$

Proof: Observe first that equation (63) is equivalent to

$$
d \Psi_{z}^{s}(\mathbb{C} \Psi(z))=\mathbb{C} z, \forall z \in V
$$

i.e. for given $z \in V$ and $\beta \in \mathbb{C}$ we can find $\delta \in \mathbb{C}$ such that $d \Psi_{z}^{s}(\beta \Psi(z))=\delta z$. Indeed by (56) and by $B_{z}(\mathbb{C} z)=\mathbb{C} z$ one has

$$
d \Psi_{z}^{s}\left(d \Psi_{z}(\mathbb{C} z)\right)=\lambda B_{z}(\mathbb{C} z)=\mathbb{C} z
$$

and by applying $\left(d \Psi_{z}^{s}\right)^{-1}$ on both sides we get (63).

In order to prove (64) let $\beta \in \mathbb{C}$ and $W$ and $S$ as in Step 3. Then for $z \in W \backslash S$ there exists $\alpha \in \mathbb{C}$ (depending on $\beta$ and $z$ ) such that $\left\langle d \Psi_{z}(\alpha z), \Psi(z)\right\rangle=\beta$. By inserting $\alpha z$ in both sides of formula (58) we obtain:

$$
\lambda^{-1} \alpha z=-f^{\prime \prime}\left(-|\Psi(z)|^{2}\right) d \Psi_{z}^{s}(\beta \Psi(z))+\lambda f^{\prime}\left(-|\Psi(z)|^{2}\right)\left(f^{\prime \prime}\left(|z|^{2}\right)\langle\alpha z, z\rangle+\alpha f^{\prime}\left(|z|^{2}\right)\right) z
$$

Hence

$$
f^{\prime \prime}\left(-|\Psi(z)|^{2}\right) d \Psi_{z}^{s}(\beta \Psi(z))=\gamma z
$$


where $\gamma=\lambda f^{\prime}\left(-|\Psi(z)|^{2}\right)\left(f^{\prime \prime}\left(|z|^{2}\right)\langle\alpha z, z\rangle+\alpha f^{\prime}\left(|z|^{2}\right)\right)-\lambda^{-1} \alpha$. Since $f$ is real analytic and $\Psi$ is a diffeomorphism $f^{\prime \prime}\left(-|\Psi(z)|^{2}\right)$ can vanish only in a discrete number of points in $W \backslash S$. Let $V \subset W$ be an open set around the origin which does not contain any of these points. We want to prove the validity of (64) in the set $V$. This is obvious for $z=0$ (since $\Psi(0)=0)$ and for all $z \in V \backslash(V \cap S)$ (this follows by (65)). So it remains to prove (64) for the points in $V \cap S \backslash\{0\}$. Let $z_{0} \in V \cap S, z_{0} \neq 0$ and $z_{n} \in V \backslash(V \cap S), z_{n} \neq 0$, be a sequence converging to $z_{0}$. Then, given $\beta \in \mathbb{C}$ there exists a sequence $\delta_{n}$ of complex numbers such that $d \Psi_{z_{n}}^{s}\left(\beta \Psi\left(z_{n}\right)\right)=\delta_{n} z_{n}$ (this follows again by (65)). By taking the limit as $n \rightarrow \infty$ the left hand side of the previous equality converges and therefore the sequence $\delta_{n}$ is forced to converge to a complex number, say $\delta_{0}$, satisfying $d \Psi_{z_{0}}^{s}\left(\beta \Psi\left(z_{0}\right)\right)=\delta_{0} z_{0}$, and we are done.

Step 5. Let $\mathcal{L} \subset \mathbb{C}^{n}$ be a complex line through the origin. Then there exists a complex line through the origin $\mathcal{L}^{*}$ such that

$$
\Psi(\mathcal{L} \cap U)=\mathcal{L}^{*} \cap U^{*}
$$

In particular $d \Psi_{0} \in U(n)$.

Proof: Let $z_{0} \in \mathcal{L}$. By the $U(n)$-invariance of $\omega_{0}, \omega, \omega^{*}$ we can assume $\Psi\left(z_{0}\right) \in \mathcal{L}$. Thus, we need to show that $\Psi(\mathcal{L} \cap U)=\mathcal{L} \cap U^{*}$. Equivalently we have to show that for every $\xi \in \mathbb{C}^{n}=\mathbb{R}^{2 n}$ orthogonal to $\mathcal{L}$, i.e. $g_{0}(z, \xi)=0$ for all $z \in \mathcal{L}$, and for every smooth curve $\gamma(t) \in \mathcal{L}$, such that $\gamma(0)=z_{0}$, one has $g_{0}(\Psi(\gamma(t)), \xi)=0$, in the interval of defintion of $\gamma(t)$, say $t \in(-a, a)$. Introduce the function $\phi_{\xi}(t)=g_{0}(\Psi(\gamma(t)), \xi)$. Then, by using Step 4, we get

$$
\frac{d \phi_{\xi}(t)}{d t}=g_{0}\left(d \Psi_{\gamma(t)}\left(\gamma^{\prime}(t)\right), \xi\right)=\beta(t) g_{0}(\Psi(\gamma(t)), \xi)=\beta(t) \phi_{\xi}(t)
$$

for some smooth function $\beta(t), t \in(-a, a)$. Then $\phi_{\xi}$ verifies a first order ordinary differential equation. Since $\phi_{\xi}(0)$ is zero then $\phi_{\xi} \equiv 0$. Thus, $\Psi(\gamma(t)) \in \mathcal{L}$ for all $t$, and this proves the first part of the step. In order to prove the last assertion notice first that $d \Psi_{0}$ is linear symplectomorphism from $\left(\mathbb{R}^{2 n}, \omega_{0}\right)$ to itself. Indeed, since $\Psi$ is a symplectic duality one has $d \Psi_{0}^{*} \omega_{0}=\left.\lambda \omega\right|_{0}=\lambda \lambda^{-1} \omega_{0}$ (the last equality is due to the second part of Step 2). Moreover, by using the first part of the present step (namely the fact that $\Psi$ sends complex lines through the origin to complex lines through the origin), a simple linear algebra argument yields $d \Psi_{0}(i v)= \pm i d \Psi_{0}(v)$, for all $v \in \mathbb{C}^{n}$. Since $d \Psi_{0}$ preserves the orientation $d \Psi_{0}(i v)=i d \Psi_{0}(v)$, for all $v \in \mathbb{C}^{n}$, and hence $d \Psi_{0} \in \operatorname{GL}(n, \mathbb{C}) \cap \operatorname{Symp}\left(\mathbb{R}^{2 n}\right)=U(n)$.

Final step. There exist an open $V \subset U$, a radial function $h: V \rightarrow \mathbb{C}$ and $A \in U(n)$ such that

$$
\Psi(z)=h(z) A z .
$$

Hence $h(z)=e^{i g(z)} \psi(z)$ where $g$ and $\psi$ are radial functions on $V$

Proof: By Step 5, $\Psi$ restricted to a suitable open subset $V \subset U$ sends complex lines through the origin (intersected with $V$ ) to complex lines through the origin (intersected with $\Psi(V)$ ). Hence there exists a complex valued function $h: V \rightarrow \mathbb{C}$ such that $\Psi(z)=h(z) d \Psi_{0}(z)$. Setting $A=d \Psi_{0} \in U(n)$ it remains to prove that $h$ is radial, i.e. it depends only on $|z|^{2}$. Since $A^{*} \omega_{0}=\omega_{0}$ and $A^{*} \omega=\omega$ we can assume that $\Psi(z)=h(z) z$.

We first show that $|h(z)|^{2}$ is radial. Equivalently we will show that $d\left(|h|^{2}\right)_{z}(v)=0$ if $v$ is a non-zero vector perpendicular to $z$, i.e., $g_{0}(z, v)=0$, for all $z \in V, z \neq 0$. Notice that this is true when $v=i z$, namely $d\left(|h|^{2}\right)_{z}(i z)=0$ for all $z \in V, z \neq 0$. Actually a strongest condition is true, namely $d h_{z}(i z)=0$ for all $z \in V \backslash\{0\}$. Indeed, if one restricts $\Psi$ to the complex line $\mathcal{L} \subset \mathbb{C}^{n}$ generated by $z$ one gets a $\lambda$-symplectic duality between $\left(\mathcal{L} \cap V,\left.\omega\right|_{\mathcal{L} \cap V)}\right.$ and $\left(\Psi(\mathcal{L} \cap V),\left.\omega^{*}\right|_{\Psi(\mathcal{L} \cap V)}\right)$ and the claim follows easily from the one-dimensional case (see Step 1 above). In order to prove our assertion for arbitrary $v$ orthogonal to $z$ we can then assume that $v$ is perpendicular to $\operatorname{span}_{\mathbb{R}}\{z, i z\}$. This means that $\omega_{0}(z, v)=\omega_{0}(i z, v)=0$. Using (51) and (52) we also get $\omega_{z}(z, v)=\omega_{z}(i z, v)=0$. Hence, on the one hand, one gets $\left(\Psi^{*} \omega_{0}\right)_{z}(i z, v)=\lambda \omega(i z, v)=0$. On the other hand,

$$
\begin{gathered}
0=\left(\Psi^{*} \omega_{0}\right)_{z}(i z, v)=\omega_{0}\left(d h_{z}(i z) z+h(z) i z, d h_{z}(v) z+h(z) v\right)= \\
=\omega_{0}\left(h(z) i z, d h_{z}(v) z+h(z) v\right)=\omega_{0}\left(h(z) i z, d h_{z}(v) z\right)+\omega_{0}(h(z) i z, h(z) v)= \\
=\omega_{0}\left(h(z) i z, d h_{z}(v) z\right)=-\operatorname{Im}\left(\left\langle h(z) i z, d h_{z}(v) z\right\rangle\right)=|z|^{2} \operatorname{Real}\left(h(z) \overline{d h_{z}(v)}\right)= \\
=\frac{|z|^{2}}{2}\left(h(z) \overline{d h_{z}(v)}+\overline{h(z)} d h_{z}(v)\right)=\frac{|z|^{2}}{2} d\left(|h|^{2}\right)_{z}(v) .
\end{gathered}
$$

It follows that $d\left(|h|^{2}\right)_{z}(v)=0$ and hence $|h|^{2}$ just depends on $|z|^{2}$. 
We now show that $h$ is radial. With the same considerations just made for $|h|^{2}$ it is enough to show that $d h_{z}(v)=0$ for all $z \in V \backslash\{0\}$ and for all $v$ perpendicular to $\operatorname{span}_{\mathbb{R}}\{z, i z\}$. For such $z$ and $v$ one has, on the one hand, $\left(\Psi^{*} \omega_{0}\right)_{z}(z, v)=\lambda \omega(z, v)=0$. On the other hand,

$$
\begin{gathered}
0=\left(\Psi^{*} \omega_{0}\right)_{z}(z, v)=|z|^{2} \frac{i}{2}(d h \wedge d \bar{h})_{z}(z, v)+\frac{i}{2} \sum_{j=1}^{n} h(z) \bar{z}_{j}\left(d z_{j} \wedge d \bar{h}\right)_{z}(z, v)+ \\
+\sum_{j=1}^{n} \frac{i}{2} z_{j} \overline{h(z)}\left(d h \wedge d \bar{z}_{j}\right)_{z}(z, v)+|h|^{2} \omega_{0}(z, v)= \\
=|z|^{2} \frac{i}{2}(d h \wedge d \bar{h})_{z}(z, v)+|z|^{2} \frac{i}{2} h(z) d \bar{h}_{z}(v)-|z|^{2} \frac{i}{2} \overline{h(z)} d h_{z}(v) .
\end{gathered}
$$

Therefore

$$
0=(d h \wedge d \bar{h})_{z}(z, v)+h(z) d \bar{h}_{z}(v)-\overline{h(z)} d h_{z}(v)=(d h \wedge d \bar{h})_{z}(z, v)-2 \overline{h(z)} d h_{z}(v)
$$

where the last equality is a consequence of the fact that we just prove that $|h|^{2}$ is radial (and hence $0=d\left(|h|^{2}\right)_{z}(v)=$ $h(z) d \bar{h}_{z}(v)+\overline{h(z)} d h_{z}(v)$ for all $z \in V \backslash\{0\}$ and for all $v$ perpendicular to $\left.\operatorname{span}_{\mathbb{R}}\{z, i z\}\right)$.

Multiplying both sides of the previous equality by $|h(z)|^{2}$ we get:

$$
\begin{gathered}
0=|h(z)|^{2} d h_{z}(z) d \bar{h}_{z}(v)-|h(z)|^{2} d h_{z}(v) d \bar{h}_{z}(z)-|h(z)|^{2} 2 \overline{h(z)} d h_{z}(v)= \\
=-\overline{h(z)} d h_{z}(z) \overline{h(z)} d h_{z}(v)-|h(z)|^{2} d h_{z}(v) d \bar{h}_{z}(z)-|h(z)|^{2} 2 \overline{h(z)} d h_{z}(v)= \\
=-\overline{h(z)} d h_{z}(v)\left(\overline{h(z)} d h_{z}(z)+h(z) d \bar{h}_{z}(z)+2|h(z)|^{2}\right)= \\
=-\overline{h(z)} d h_{z}(v)\left(d\left(|h|^{2}\right)_{z}(z)+2|h(z)|^{2}\right) .
\end{gathered}
$$

Note that $h(z) \neq 0$ for $z \neq 0$ since the map $\Psi: V \rightarrow \Psi(V), z \mapsto h(z) z$ is injective (it is a diffeomorphism). Hence, in order to show that $h$ is a radial function it is enough to prove that $d\left(|h|^{2}\right)_{z}(z)+2|h(z)|^{2}$ cannot vanish on any open subset of $V \backslash\{0\}$. Since $|h|^{2}$ is radial we can restrict the problem to the real line $\mathbb{R} e_{1}, e_{1}=(1,0, \ldots, 0)$. More precisely, by defining $\sigma(t)=\left|h\left(t e_{1}\right)\right|^{2}$ and $I=\left\{t \in \mathbb{R} \mid t \sigma^{\prime}(t)+2 \sigma(t)=0\right\}$ the radiality of $h$ will be guaranteed if $I$ does not contain any open subset of the real line. Assume, by contradiction, that there exists such an open subset. Then in this set $\sigma$ solves the differential equation $t \sigma^{\prime}(t)+2 \sigma(t)=0$ and so $\sigma(t)=\frac{1}{(c t)^{2}}$ for some real constant $c$. This is the desired contradiction since $|h(0)|^{2}=\sigma(0)$ is a well defined real number.

An immediate consequence of Theorem [1.6] is the following corollary which can be considered a generalization of the second part of Theorem 1.1.

Corollary 3.3 Let $\Psi: U \rightarrow U^{*}$ be a $\lambda$-symplectic duality between two radial forms $\omega$ and $\omega^{*}$. Then there exists an open subset $V \subset U$ where the restriction of $\Psi$ takes complex and totally geodesic submanifolds through the origin of $(V, \omega)$ to complex and totally geodesic submanifolds through the origin of $\left(\Psi(V), \omega_{0}\right)$.

Proof: Let $V \subset U \subset \mathbb{C}^{n}$ be an open subset containing the origin such that the restriction of $\Psi$ to $V$ is of the form (11), i.e. $\Psi(z)=e^{i g(z)} \psi(z) A(z), z \in V$. Since $\omega$ is radial it is easy to see that a complex and totally geodesic submanifold of $(V, \omega)$ of (complex) dimension $k$ is the intersection of $V$ with a $k$-dimensional linear subspace of $\mathbb{C}^{n}$. Therefore $\Psi(V \cap T)=T^{*} \cap \Psi(V)$ where $T^{*}$ is the $k$-dimensional space of $\mathbb{C}^{n}$ given by $A(T)$.

\section{Applications and examples}

In this section we provide some examples and applications of our results. The first two subsections deal with Hartogs domains and the Taub-NUT metric respectively which are important examples in the rotation invariant case. In the third subsection, where we consider the radial case, we exhibit an example of radial Kähler form (different from the hyperbolic metric) for which there exists a $\lambda$-symplectic duality. In all this section given a rotation invariant (or even radial) Kähler form $\omega=\frac{i}{2} \partial \bar{\partial} \Phi$ (on an open subset of $\mathbb{C}^{n}$ containing the origin) we say that it admits a $\lambda$-symplectic duality if there exists a $\lambda$-symplectic duality $\Psi: U \rightarrow U^{*}$ between $\omega$ and $\omega^{*}=\frac{i}{2} \partial \bar{\partial} \Phi^{*}$, where $\Phi^{*}$ is the (local) dual of $\Phi$ (defined on a suitable neighborhood $M^{*}$ of the origin). 


\subsection{Hartogs domains}

Let $x_{0} \in \mathbb{R}^{+} \cup\{+\infty\}$ and let $F:\left[0, x_{0}\right) \rightarrow(0,+\infty)$ be a decreasing real analytic function, on $\left(0, x_{0}\right)$. The Hartogs domain $D_{F} \subset \mathbb{C}^{n}$ associated to the function $F$ is defined by

$$
D_{F}=\left\{\left.\left(z_{0}, z_{1}, \ldots, z_{n-1}\right) \in \mathbb{C}^{n}|| z_{0}\right|^{2}<x_{0},\left|z_{1}\right|^{2}+\cdots+\left|z_{n-1}\right|^{2}<F\left(\left|z_{0}\right|^{2}\right)\right\} .
$$

One can prove that, under the assumption $-\left(\frac{x F^{\prime}(x)}{F(x)}\right)^{\prime}>0$ for every $x \in\left[0, x_{0}\right)$, the natural $(1,1)$-form on $D_{F}$ given by

$$
\omega_{F}=\frac{i}{2} \partial \bar{\partial} \log \frac{1}{F\left(\left|z_{0}\right|^{2}\right)-\left|z_{1}\right|^{2}-\cdots-\left|z_{n-1}\right|^{2}}
$$

is a Kähler form on $D_{F}$. The previous equality is equivalent to the strongly pseudoconvexity of $D_{F}$ (see [5] for a proof and also [6, [8, [12] and [9] for other properties of these domains).

Notice that, when $x_{0}=1$ and $F(x)=1-x$, then the corresponding Hartogs domain is the $n$-dimensional unit ball endowed with the hyperbolic form $\omega_{\text {hyp }}$. In this case, we have already observed in the introduction that $\omega_{h y p}$ admits a special $\lambda$-symplectic duality. We now prove that in fact this is the only case among Hartogs domains, namely If $\left(D_{F}, \omega_{F}\right)$ admits a $\lambda$-symplectic duality then $\left(D_{F}, \omega_{F}\right)$ is holomorphically isometric to an open subset of the complex hyperbolic space. In order to prove our assertion notice first that the potential for the Kähler form $\omega_{F}$ is rotation invariant and has $\tilde{\Phi}\left(x_{0}, x_{1}, \ldots, x_{n-1}\right)=-\log \left(F\left(x_{0}\right)-\sum_{j=1}^{n-1} x_{j}\right)$ as associated function. Therefore by Theorem 1.5 (cf. equations (10) $)\left(D_{F}, \omega_{F}\right)$ admits a $\lambda$-symplectic duality iff the following two equations are satisfied on a neighbourhood of the origin of $\mathbb{R}^{n}$ :

$$
\lambda^{2} \frac{F^{\prime}\left(x_{0}\right)}{F\left(x_{0}\right)-\sum_{j=1}^{n-1} x_{j}} \cdot \frac{F^{\prime}\left(-\lambda \frac{\partial \tilde{\Phi}}{\partial x_{0}} x_{0}\right)}{F\left(-\lambda \frac{\partial \tilde{\Phi}}{\partial x_{0}} x_{0}\right)+\sum_{j=1}^{n-1} \lambda \frac{\partial \tilde{\Phi}}{\partial x_{j}} x_{j}}=1
$$

and

$$
\frac{1}{F\left(x_{0}\right)-\sum_{j=1}^{n-1} x_{j}} \cdot \frac{1}{F\left(-\lambda \frac{\partial \tilde{\Phi}}{\partial x_{0}} x_{0}\right)+\sum_{j=1}^{n-1} \lambda \frac{\partial \tilde{\Phi}}{\partial x_{j}} x_{j}}=1 .
$$

Substituting the second one into the first one we get

$$
\lambda^{2} F^{\prime}\left(x_{0}\right) \cdot F^{\prime}\left(\frac{\lambda x_{0} F^{\prime}\left(x_{0}\right)}{F\left(x_{0}\right)-\sum_{j=1}^{n-1} x_{j}}\right)=1 .
$$

If we fix $x_{0}$ in this equation and let $t=\sum_{j=1}^{n-1} x_{j}$ take values in a small open interval contained in $\left[0, F\left(x_{0}\right)\right)$, we get that $F^{\prime}(x)$ is constant on a sufficiently small interval, and hence $F^{\prime}$ is constant. So $F(x)=c_{1}-c_{2} x$ for some $c_{1}, c_{2}>0$, which implies that $D_{F}$ is holomorphically isometric to an open subset of the hyperbolic space $\mathbb{C} H^{n}$ via the $\operatorname{map} \phi: D_{F} \rightarrow \mathbb{C} H^{n},\left(z_{0}, z_{1}, \ldots, z_{n-1}\right) \mapsto\left(\frac{z_{0}}{\sqrt{c_{1} / c_{2}}}, \frac{z_{1}}{\sqrt{c_{1}}}, \ldots, \frac{z_{n-1}}{\sqrt{c_{1}}}\right)$.

\subsection{The Taub-NUT metric}

In [7. C. LeBrun constructed the following family of Kähler forms on $\mathbb{C}^{2}$ defined by $\omega_{m}=\frac{i}{2} \partial \bar{\partial} \Phi_{m}$, where

$$
\Phi_{m}(U, V)=U+V+m\left(U^{2}+V^{2}\right), m \geq 0
$$

and $U$ and $V$ are implicitly defined by

$$
\left|z_{1}\right|^{2}=e^{2 m(U-V)} U,\left|z_{2}\right|^{2}=e^{2 m(V-U)} V .
$$

For $m=0$ one gets the flat metric, while for $m>0$ each of the metrics of this family represents the first example of complete Ricci flat (non-flat) metric on $\mathbb{C}^{2}$ having the same volume form of the flat metric $\omega_{0}$. Moreover, for $m>0$, these metrics are isometric (up to dilation and rescaling) to the Taub-NUT metric. In [1] it is proven that $\left(\mathbb{C}^{2}, \omega_{m}\right)$ is globally symplectomorphic to $\left(\mathbb{R}^{4}, \omega_{0}\right)$ via a special symplectic map. 
We claim that there exists a special $\lambda$-symplectic duality $\Psi$ for $\left(\mathbb{C}^{2}, \omega_{m}\right)$ if and only if $m=0$. In order to prove our claim let $x_{j}=\left|z_{j}\right|^{2}, j=1,2$. By the inverse function theorem one easily gets

$$
\frac{\partial \tilde{\Phi}_{m}}{\partial x_{1}}=(1+2 m V) e^{2 m(V-U)}, \quad \frac{\partial \tilde{\Phi}_{m}}{\partial x_{2}}=(1+2 m U) e^{2 m(U-V)}
$$

so that $\frac{\partial \tilde{\Phi}_{m}}{\partial x_{1}} x_{1}=U+2 m x_{1} x_{2}$ and $\frac{\partial \tilde{\Phi}_{m}}{\partial x_{2}} x_{2}=V+2 m x_{1} x_{2}$. Equations (10) for $x_{2}=0$ write respectively

$$
\begin{gathered}
\lambda^{2} e^{-2 m U\left(x_{1}, 0\right)} e^{-2 m U\left(-\lambda U\left(x_{1}, 0\right), 0\right)}=1 \\
\lambda^{2}\left(1+2 m U\left(x_{1}, 0\right)\right) e^{2 m U\left(x_{1}, 0\right)}\left(1+2 m U\left(-\lambda U\left(x_{1}, 0\right), 0\right)\right) e^{2 m U\left(-\lambda U\left(x_{1}, 0\right), 0\right)}=1 .
\end{gathered}
$$

By the first one we get

$$
U\left(-\lambda U\left(x_{1}, 0\right), 0\right)=\frac{1}{m} \log \lambda-U\left(x_{1}, 0\right)
$$

which, replaced in the second one, gives

$$
\lambda^{4}\left(1+2 m U\left(x_{1}, 0\right)\right)\left(1+2 \log \lambda-2 m U\left(x_{1}, 0\right)\right)=1 .
$$

The latter is a polynomial equation of degree 2 in $2 m U\left(x_{1}, 0\right)$. Let $m \neq 0$. Then, either this equation has not solution, and we are done, or it implies that $U\left(x_{1}, 0\right)$ is constant in a neighbourhood of 0 . But in this case, since $x_{1}=e^{2 m U} U$, also $x_{1}$ must be constant, which is impossible. This proves our claim.

\subsection{Examples in the radial case}

Let $M$ be an open neighbourhood of 0 in $\mathbb{C}^{n}$, endowed with a radial Kähler form $\omega=\frac{i}{2} \partial \bar{\partial} f$, with $f=f\left(|z|^{2}\right)$. In this case, we know that the existence of a radial invariant $\lambda$-symplectic duality is guaranteed by equation (13), namely $\lambda^{2} f^{\prime}(x) f^{\prime}\left(-\lambda x f^{\prime}(x)\right)=1$ on a suitable neighbourhood of the origin of $\mathbb{R}$.

It is easy to see (in accordance with what we already knew) that $f(x)=\frac{1}{\lambda} x$ (the flat metric), $f(x)=-\frac{1}{\lambda} \log (1-x)$ (the hyperbolic metric) and $f(x)=\frac{1}{\lambda} \log (1+x)$ (the Fubini-Study metric) satisfy this equation. In order to see other solutions different from these cases, notice that (13) can be rewritten as

$$
G(G(x))=x
$$

where $G(x)=-\lambda x f^{\prime}(x)$. Thus if the graph of $y=G(x)$ is symmetric with respect to the straight line $y=x$, then $G(x)$ satisfies (67). Take for example

$$
G(x)=-\frac{\sqrt{2}}{2}+x+\frac{1}{2} \sqrt{2-8 \sqrt{2} x}
$$

which is defined in a neighbourhood of 0 , satisfies this condition (we obtained this function by simply rotating clockwise the graph of the even function $y=-x^{2}$ by an angle of $\pi / 4$ ). Notice also that this $G$ is analytic in 0 and satisfies $G(0)=0$, so that $G(x) / x$ is also analytic. Then, by integrating $f^{\prime}(x)=-G(x) / \lambda x$, we get a function $f(x)$ which satisfies the equation of symplectic duality and such that $f^{\prime}(0)=-\frac{G^{\prime}(0)}{\lambda}=\frac{1}{\lambda}>0$, so that it defines a Kähler metric in a sufficiently small neighbourhood of the origin. A simple calculation shows that the Kähler metric associated to this potential $f$ has not constant curvature and so this yields a Kähler metric which admits a $\lambda$-symplectic duality but which does not have constant curvature.

Finally, an easy example of potential which admits a local dual but it does not admit a $\lambda$-symplectic duality is given by $f(x)=x-\frac{x^{2}}{4}, x=|z|^{2}$, in a suitable neighbourhood of the origin.

\section{$5 \quad$ Appendix}

The following lemma provides necessary and sufficient conditions for a given rotation invariant special map to be symplectic. 
Lemma 5.1 Let $C \subseteq \mathbb{C}^{n}$ and $S \subseteq \mathbb{C}^{n}$ be two complex domains containing the origin endowed with rotation invariant Kähler potentials $\alpha$ and $\beta$ and corresponding Kähler forms $\omega_{\alpha}=\frac{i}{2} \partial \bar{\partial} \alpha$ and $\omega_{\beta}=\frac{i}{2} \partial \bar{\partial} \beta$ respectively. Then a rotation invariant special map $\Psi: C \rightarrow S$ satisfies $\Psi^{*}\left(\omega_{\beta}\right)=\omega_{\alpha}$ if and only if

$$
\tilde{\psi}_{k}^{2} \frac{\partial \tilde{\beta}}{\partial x_{k}}\left(\tilde{\psi}_{1}^{2} x_{1}, \ldots, \tilde{\psi}_{n}^{2} x_{n}\right)=\frac{\partial \tilde{\alpha}}{\partial x_{k}}, \quad k=1, \ldots, n,
$$

where $\tilde{\alpha}: \tilde{C} \subset \mathbb{R}^{n} \rightarrow \mathbb{R}$ (resp. $\tilde{\beta}: \tilde{S} \subset \mathbb{R}^{n} \rightarrow \mathbb{R}$ ) is the function associated to $\alpha$ (resp. $\beta$ ) (see Section $Q$ for the definition of special maps).

Proof: Since

$$
\omega_{\beta}=\frac{i}{2} \sum_{i, j=1}^{n}\left(\frac{\partial^{2} \tilde{\beta}}{\partial x_{i} \partial x_{j}} \bar{z}_{j} z_{i}+\frac{\partial \tilde{\beta}}{\partial x_{i}} \delta_{i j}\right) d z_{j} \wedge d \bar{z}_{i}
$$

one gets

$$
\Psi^{*}\left(\omega_{\beta}\right)=\frac{i}{2} \sum_{i, j=1}^{n}\left(\frac{\partial^{2} \tilde{\beta}}{\partial x_{i} \partial x_{j}}(\Psi) \Psi_{i} \bar{\Psi}_{j}+\frac{\partial \tilde{\beta}}{\partial x_{j}}(\Psi) \delta_{i j}\right) d \Psi_{j} \wedge d \bar{\Psi}_{i}
$$

where $\frac{\partial \tilde{\beta}}{\partial x_{j}}(\Psi)=\frac{\partial \tilde{\beta}}{\partial x_{j}}\left(\tilde{\psi}_{1}^{2} x_{1}, \ldots, \tilde{\psi}_{n}^{2} x_{n}\right)$ and $\frac{\partial^{2} \tilde{\beta}}{\partial x_{i} \partial x_{j}}(\Psi)=\frac{\partial^{2} \tilde{\beta}}{\partial x_{i} \partial x_{j}}\left(\tilde{\psi}_{1}^{2} x_{1}, \ldots, \tilde{\psi}_{n}^{2} x_{n}\right)$.

If one denotes by

$$
\Psi^{*}\left(\omega_{\beta}\right)=\Psi^{*}\left(\omega_{\beta}\right)_{(2,0)}+\Psi^{*}\left(\omega_{\beta}\right)_{(1,1)}+\Psi^{*}\left(\omega_{\beta}\right)_{(0,2)}
$$

the decomposition of $\Psi^{*}\left(\omega_{\beta}\right)$ into addenda of type $(2,0),(1,1)$ and $(0,2)$ one has:

$$
\begin{gathered}
\Psi^{*}\left(\omega_{\beta}\right)_{(2,0)}=\frac{i}{2} \sum_{i, j, k, l=1}^{n}\left(\frac{\partial^{2} \tilde{\beta}}{\partial x_{i} \partial x_{j}}(\Psi) \Psi_{i} \bar{\Psi}_{j}+\frac{\partial \tilde{\beta}}{\partial x_{j}}(\Psi) \delta_{i j}\right) \frac{\partial \Psi_{j}}{\partial z_{k}} \frac{\partial \bar{\Psi}_{i}}{\partial z_{l}} d z_{k} \wedge d z_{l} \\
\Psi^{*}\left(\omega_{\beta}\right)_{(1,1)}=\frac{i}{2} \sum_{i, j, k, l=1}^{n}\left(\frac{\partial^{2} \tilde{\beta}}{\partial x_{i} \partial x_{j}}(\Psi) \Psi_{i} \bar{\Psi}_{j}+\frac{\partial \tilde{\beta}}{\partial x_{j}}(\Psi) \delta_{i j}\right)\left(\frac{\partial \Psi_{j}}{\partial z_{k}} \frac{\partial \bar{\Psi}_{i}}{\partial \bar{z}_{l}}-\frac{\partial \Psi_{j}}{\partial \bar{z}_{l}} \frac{\partial \bar{\Psi}_{i}}{\partial z_{k}}\right) d z_{k} \wedge d \bar{z}_{l} \\
\Psi^{*}\left(\omega_{\beta}\right)_{(0,2)}=\frac{i}{2} \sum_{i, j, k, l=1}^{n}\left(\frac{\partial^{2} \tilde{\beta}}{\partial x_{i} \partial x_{j}}(\Psi) \Psi_{i} \bar{\Psi}_{j}+\frac{\partial \tilde{\beta}}{\partial x_{j}}(\Psi) \delta_{i j}\right) \frac{\partial \Psi_{j}}{\partial \bar{z}_{k}} \frac{\partial \bar{\Psi}_{i}}{\partial \bar{z}_{l}} d \bar{z}_{k} \wedge d \bar{z}_{l} .
\end{gathered}
$$

Since $\Psi_{j}(z)=\tilde{\psi}_{j}\left(\left|z_{1}\right|^{2}, \ldots,\left|z_{n}\right|^{2}\right) z_{j}$, one has:

$$
\frac{\partial \Psi_{i}}{\partial z_{k}}=\frac{\partial \tilde{\psi}_{i}}{\partial x_{k}} z_{i} \bar{z}_{k}+\tilde{\psi}_{i} \delta_{i k}, \frac{\partial \Psi_{i}}{\partial \bar{z}_{k}}=\frac{\partial \tilde{\psi}_{i}}{\partial x_{k}} z_{k} z_{i}
$$

and

$$
\frac{\partial \bar{\Psi}_{i}}{\partial \bar{z}_{k}}=\frac{\partial \tilde{\psi}_{i}}{\partial x_{k}} z_{k} \bar{z}_{i}+\tilde{\psi}_{i} \delta_{i k}, \frac{\partial \bar{\Psi}_{i}}{\partial z_{k}}=\frac{\partial \tilde{\psi}_{i}}{\partial x_{k}} \bar{z}_{k} \bar{z}_{i}
$$

By inserting (72) and (73) into (69) and (70) after a long, but straightforward computation, one obtains:

$$
\Psi^{*}\left(\omega_{\beta}\right)_{(2,0)}=\frac{i}{2} \sum_{k, l=1}^{n} \frac{A_{k l}}{2} \bar{z}_{k} \bar{z}_{l} d z_{k} \wedge d z_{l}
$$

and

$$
\Psi^{*}\left(\omega_{\beta}\right)_{(1,1)}=\frac{i}{2} \sum_{k, l=1}^{n}\left[\left(\frac{A_{k l}+A_{l k}}{2}+\frac{\partial^{2} \tilde{\beta}}{\partial x_{k} \partial x_{l}}(\Psi) \tilde{\psi}_{k}^{2} \tilde{\psi}_{l}^{2}\right) \bar{z}_{k} z_{l}+\frac{\partial \tilde{\beta}}{\partial x_{k}}(\Psi) \delta_{k l} \tilde{\psi}_{k}^{2}\right] d z_{k} \wedge d \bar{z}_{l}
$$

where

$$
A_{k l}=\frac{\partial \tilde{\beta}}{\partial x_{k}}(\Psi) \frac{\partial \tilde{\psi}_{k}^{2}}{\partial x_{l}}+\tilde{\psi}_{k}^{2} \sum_{j=1}^{n} \frac{\partial^{2} \tilde{\beta}}{\partial x_{j} \partial x_{k}}(\Psi) \frac{\partial \tilde{\psi}_{j}^{2}}{\partial x_{l}}\left|z_{j}\right|^{2}
$$


Now, we assume that

$$
\Psi^{*}\left(\omega_{\beta}\right)=\omega_{\alpha}=\frac{i}{2} \sum_{k, l=1}^{n}\left(\frac{\partial^{2} \tilde{\alpha}}{\partial x_{k} \partial x_{l}} \overline{z_{k}} z_{l}+\frac{\partial \tilde{\alpha}}{\partial x_{l}} \delta_{l k}\right) d z_{k} \wedge d \bar{z}_{l} .
$$

Then the terms $\Psi^{*}\left(\omega_{\beta}\right)_{(2,0)}$ and $\Psi^{*}\left(\omega_{\beta}\right)_{(0,2)}$ are equal to zero. This is equivalent to the fact that (176) is symmetric in $k, l$.

Hence, by setting

$$
\Gamma_{l}=\tilde{\psi}_{l}^{2} \frac{\partial \tilde{\beta}}{\partial x_{l}}(\Psi), l=1, \ldots, n
$$

equation (75) becomes

$$
\begin{gathered}
\Psi^{*}\left(\omega_{\beta}\right)_{(1,1)}=\frac{i}{2} \sum_{k, l=1}^{n}\left[\left(A_{k l}+\frac{\partial^{2} \tilde{\beta}}{\partial x_{k} \partial x_{l}}(\Psi) \tilde{\psi}_{k}^{2} \tilde{\psi}_{l}^{2}\right) \bar{z}_{k} z_{l}+\frac{\partial \tilde{\beta}}{\partial x_{k}}(\Psi) \delta_{k l} \tilde{\psi}_{k}^{2}\right] d z_{k} \wedge d \bar{z}_{l}= \\
=\frac{i}{2} \sum_{k, l=1}^{n}\left(\frac{\partial \Gamma_{l}}{\partial x_{k}} \overline{z_{k}} z_{l}+\Gamma_{k} \delta_{k l}\right) d z_{k} \wedge d \bar{z}_{l} .
\end{gathered}
$$

So, $\Psi^{*}\left(\omega_{\beta}\right)=\omega_{\alpha}$ implies

$$
\frac{i}{2} \sum_{k, l=1}^{n}\left(\frac{\partial \Gamma_{l}}{\partial x_{k}} \overline{z_{k}} z_{l}+\Gamma_{k} \delta_{l k}\right) d z_{k} \wedge d \bar{z}_{l}=\frac{i}{2} \sum_{k, l=1}^{n}\left(\frac{\partial^{2} \tilde{\alpha}}{\partial x_{k} \partial x_{l}} \overline{z_{k}} z_{l}+\frac{\partial \tilde{\alpha}}{\partial x_{l}} \delta_{k l}\right) d z_{k} \wedge d \bar{z}_{l} .
$$

In this equality, we distinguish the cases $l \neq k$ and $l=k$ and get respectively

$$
\frac{\partial \Gamma_{l}}{\partial x_{k}}=\frac{\partial^{2} \tilde{\alpha}}{\partial x_{k} \partial x_{l}} \quad(k \neq l)
$$

and

$$
\frac{\partial \Gamma_{k}}{\partial x_{k}} x_{k}+\Gamma_{k}=\frac{\partial^{2} \tilde{\alpha}}{\partial x_{k}^{2}} x_{k}+\frac{\partial \tilde{\alpha}}{\partial x_{k}} .
$$

By defining $A_{k}=\Gamma_{k}-\frac{\partial \tilde{\alpha}}{\partial x_{k}}$, these equations become respectively

$$
\frac{\partial A_{k}}{\partial x_{l}}=0 \quad(l \neq k)
$$

and

$$
\frac{\partial A_{k}}{\partial x_{k}} x_{k}=-A_{k}
$$

The first equation implies that $A_{k}$ does not depend on $x_{l}$ and so by the second one we have

$$
A_{k}=\Gamma_{k}-\frac{\partial \tilde{\alpha}}{\partial x_{k}}=\frac{c_{k}}{x_{k}}
$$

for some constant $c_{k} \in \mathbb{R}$. Since the domains contains the origin this forces $c_{k}=0, \forall k$, and hence, by (77), we get

$$
\Gamma_{k}=\tilde{\psi}_{k}^{2} \frac{\partial \tilde{\beta}}{\partial x_{k}}(\Psi)=\frac{\partial \tilde{\beta}}{\partial x_{k}}\left(\tilde{\psi}_{1}^{2} x_{1}, \ldots, \tilde{\psi}_{n}^{2} x_{n}\right)=\frac{\partial \tilde{\alpha}}{\partial x_{k}}, \quad k=1, \ldots, n
$$

namely (68).

In order to prove the converse of Lemma 5.1, notice that by differentiating (68) with respect to $l$ one gets:

$$
\frac{\partial^{2} \tilde{\alpha}}{\partial x_{k} \partial x_{l}}=A_{k l}+\frac{\partial^{2} \tilde{\beta}}{\partial x_{k} \partial x_{l}} \tilde{\psi}_{k}^{2} \tilde{\psi}_{l}^{2}
$$

with $A_{k l}$ given by (76). By $\frac{\partial^{2} \tilde{\alpha}}{\partial x_{k} \partial x_{l}}=\frac{\partial^{2} \tilde{\alpha}}{\partial x_{l} \partial x_{k}}$ and $\frac{\partial^{2} \tilde{\beta}}{\partial x_{k} \partial x_{l}} \tilde{\psi}_{k}^{2} \tilde{\psi}_{l}^{2}=\frac{\partial^{2} \tilde{\beta}}{\partial x_{l} \partial x_{k}} \tilde{\psi}_{l}^{2} \tilde{\psi}_{k}^{2}$ one gets $A_{k l}=A_{l k}$. Then, by (74), the addenda of type $(2,0)$ (and $(0,2))$ in $\Psi^{*}\left(\omega_{\beta}\right)$ vanish. Moreover, by (76) and (78), it follows that $\Psi^{*}\left(\omega_{\beta}\right)=\omega_{\alpha}$. 


\section{References}

[1] F. Cuccu and A. Loi, Global symplectic coordinates on complex domains, J. Geom. and Phys. 56 (2006), 247-259.

[2] A. J. Di Scala and A. Loi, Kähler maps of hermitian symmetric spaces into complex space forms, Geom. Dedicata 125 (2007), 103-113.

[3] A. J. Di Scala and A. Loi, Symplectic duality of symmetric spaces, to appear in Adv. Math.

[4] A. J. Di Scala, A. Loi and G. Roos, The bisymplectomorphism group of a bounded symmetric domain, to appear in Transformation Groups.

[5] A. J. Di Scala, A. Loi and F. Zuddas, Riemannian geometry of Hartogs domains, to appear in International Journal of Mathematics.

[6] M. Engliš, Berezin Quantization and Reproducing Kernels on Complex Domains, Trans. Amer. Math. Soc. vol. 348 (1996), 411-479.

[7] C. LeBrun, Complete Ricci-flat Kähler metrics on $\mathbb{C}^{n}$ need not be flat, Proceedings of Symposia in Pure Mathematics, vol. 52 (1991), Part 2, 297-304.

[8] A. Loi, Holomorphic maps of Hartogs domains into complex space forms, Riv. Mat. Univ. Parma (7) vol. 1 (2002), 103-113.

[9] A. Loi, Regular quantizations of Kähler manifolds and constant scalar curvature metrics, Journal of Geometry and Physics 53 (2005), 354-364.

[10] A. Loi, Calabi's diastasis function for Hermitian symmetric spaces, Differential Geom. Appl. 24 (2006), no. 3, 311-319.

[11] A. Loi and F. Zuddas, Symplectic maps of complex domains into complex space forms, to appear in J. Geom. Phys.

[12] A. Loi and F. Zuddas, Extremal metrics on Hartogs domains, arXiv:0705.2124,

Università di Torino,

E-mail: antonio.discala@polito.it

Università di Cagliari,

E-mail: loi@unica.it

Università di Cagliari,

E-mail: fzuddas@unica.it 